Supporting information for:

\title{
A New Class of Versatile Chiral-Bridged Atropisomeric Diphosphine Ligands: Remarkably Efficient Ligand Syntheses and Their Applications in Highly Enantioselective Hydrogenation Reactions
}

Liqin Qiu, Fuk Yee Kwong, Jing Wu, Wai Har Lam, Shusun Chan, Wing-Yiu Yu, Rongwei Guo, Zhongyuan Zhou, Albert S. C. Chan*

Open laboratory of Chirotechnology of the Institute of Molecular Technology for Drug

Discovery and Synthesis and Department of Applied Biology and Chemical Technology, The Hong Kong Polytechnic University, Hong Kong 
Synthesis of $(2 S, 5 S)$-hexanediol-ditosylate $(2 \mathrm{c})$. Under $\mathrm{N}_{2}$ atmosphere and at $0{ }^{\circ} \mathrm{C}$, a solution of $(2 S, 5 S)$-hexanediol $(4.0 \mathrm{~g}, 33.8 \mathrm{mmol})$ in $20 \mathrm{~mL}$ pyridine was added slowly into a mixture of $p$-toluenesulfonyl chloride $(14.20 \mathrm{~g}, 74.5 \mathrm{mmol})$ in $30 \mathrm{~mL}$ of pyridine over a period of 4 hours. The reaction temperature was kept at $0{ }^{\circ} \mathrm{C}$ for $2 \mathrm{hr}$, then it was raised to room temperature and the reaction was continued overnight. The resultant thick mixture was poured into ice-water $(500 \mathrm{~mL})$ with vigorous stirring as the product precipitated out. After stirring for an hour, it was filtered cold and the solid was washed with $500 \mathrm{~mL}$ of cold water and sucked dry. After drying further in vacuo overnight, the pink raw product was recrystallized from acetone to afford pure white crystals 2c (10.331g, $24.2 \mathrm{mmol}, 71.7 \%$ yield). ${ }^{1} \mathrm{H} \mathrm{NMR}\left(\mathrm{CDCl}_{3}\right) 500 \mathrm{MHz}: \delta 1.13(\mathrm{~d}, J=6.5 \mathrm{~Hz}$, $6 \mathrm{H}), 1.48-1.51(\mathrm{~m}, 2 \mathrm{H}), 1.56-1.61(\mathrm{~m}, 2 \mathrm{H}), 2.45(\mathrm{~s}, 6 \mathrm{H}), 4.50-4.53(\mathrm{~m}, 2 \mathrm{H}), 7.34(\mathrm{~d}, J=8$ $\mathrm{Hz}, 4 \mathrm{H}), 7.77(\mathrm{~d}, J=8.5 \mathrm{~Hz}, 4 \mathrm{H}) ;{ }^{13} \mathrm{C} \mathrm{NMR}\left(\mathrm{CDCl}_{3}\right) 125 \mathrm{MHz}: \delta 20.77,21.58,31.55$, 79.01, 127.65, 129.79, 134.23, 144.65; MS (ESI): calcd for $\mathrm{C}_{20} \mathrm{H}_{26} \mathrm{~S}_{2} \mathrm{O}_{6}[\mathrm{M}]^{+} 426.5$, found 427; HRMS (ESI): calcd for $\mathrm{C}_{20} \mathrm{H}_{26} \mathrm{~S}_{2} \mathrm{O}_{6}[\mathrm{M}+\mathrm{Na}]^{+}$449.1068, found 449.1109; calcd for $\mathrm{C}_{20} \mathrm{H}_{27} \mathrm{~S}_{2} \mathrm{O}_{6}[\mathrm{M}+\mathrm{H}]^{+} 427.1249$, found 427.1295; $[\alpha]^{20}{ }_{\mathrm{D}}=-14.7^{\circ}\left(c=1, \mathrm{CHCl}_{3}\right)$. 
${ }^{1} \mathrm{H}$ NMR (500 MHz, $\mathrm{CDCl}_{3}$ ) of $\mathbf{2 c}$
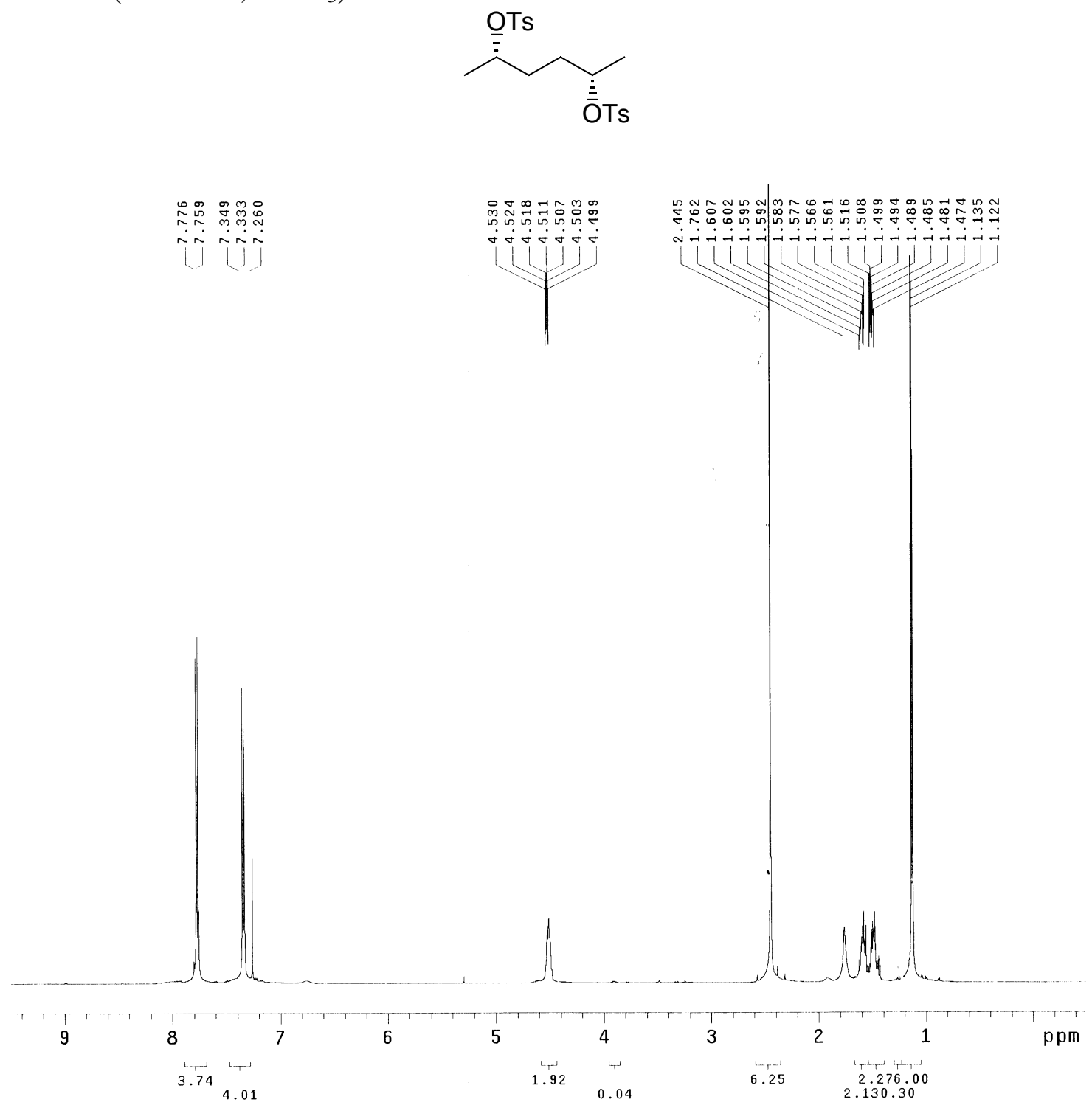
${ }^{13} \mathrm{C}$ NMR (125 MHz, $\mathrm{CDCl}_{3}$ ) of $\mathbf{2 c}$
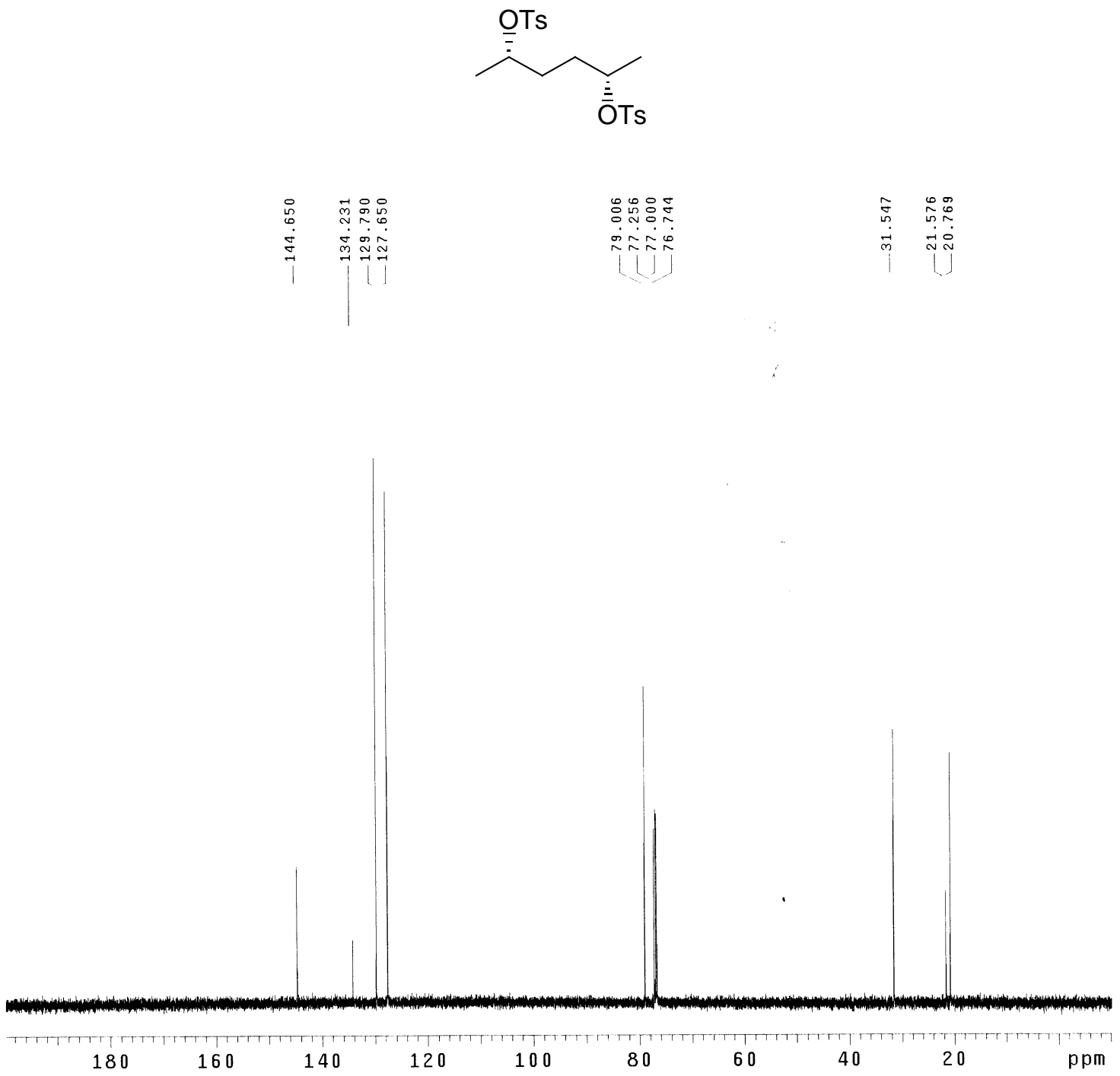
${ }^{1} \mathrm{H}$ NMR $\left(500 \mathrm{MHz}, \mathrm{CDCl}_{3}\right)$ of $\mathbf{3 b}$

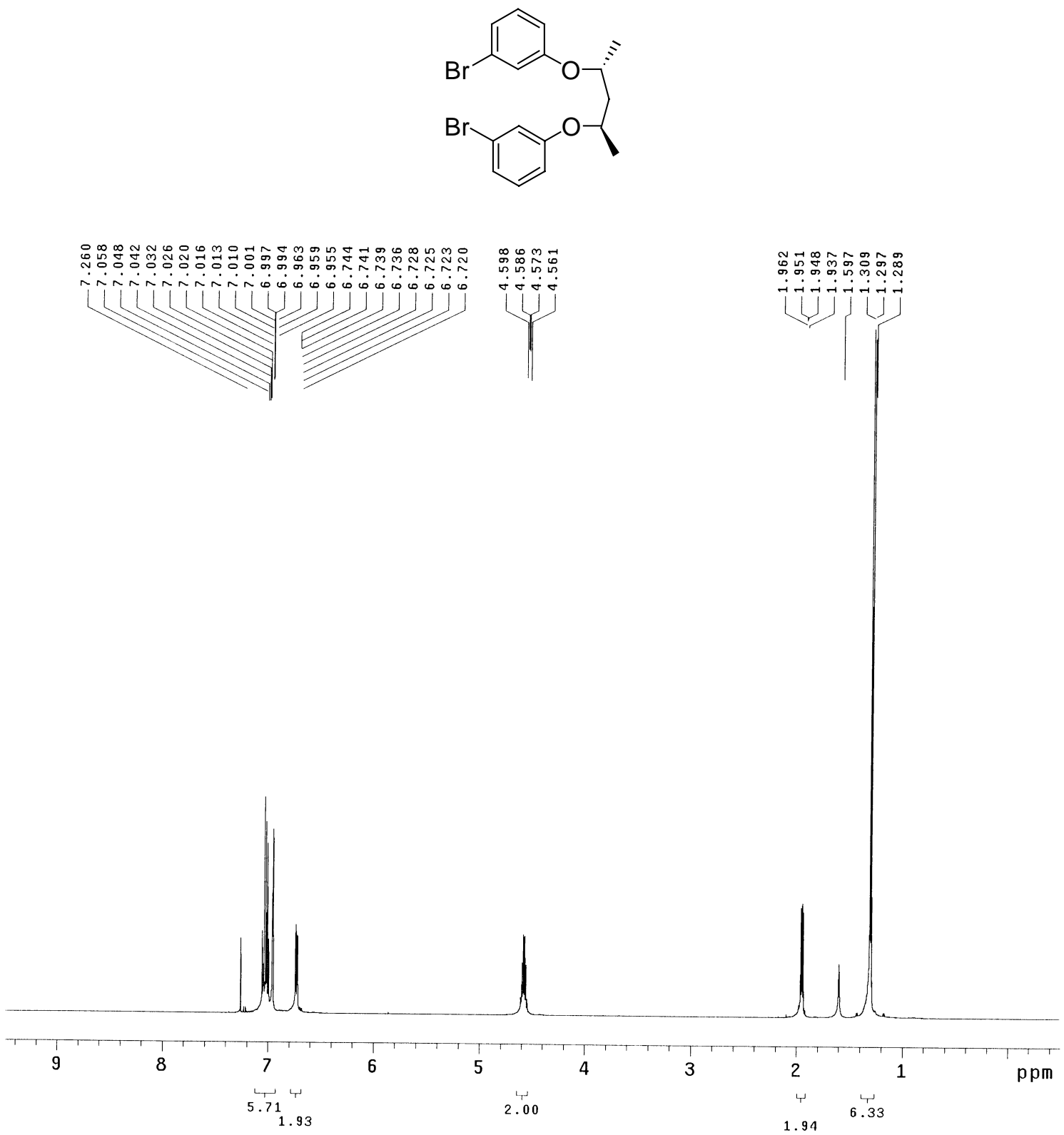


${ }^{13} \mathrm{C}$ NMR $\left(125 \mathrm{MHz}, \mathrm{CDCl}_{3}\right)$ of $\mathbf{3 b}$<smiles>CC(C)CC(C)Oc1cccc(Br)c1</smiles>

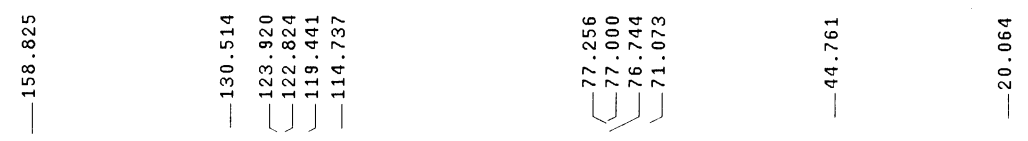

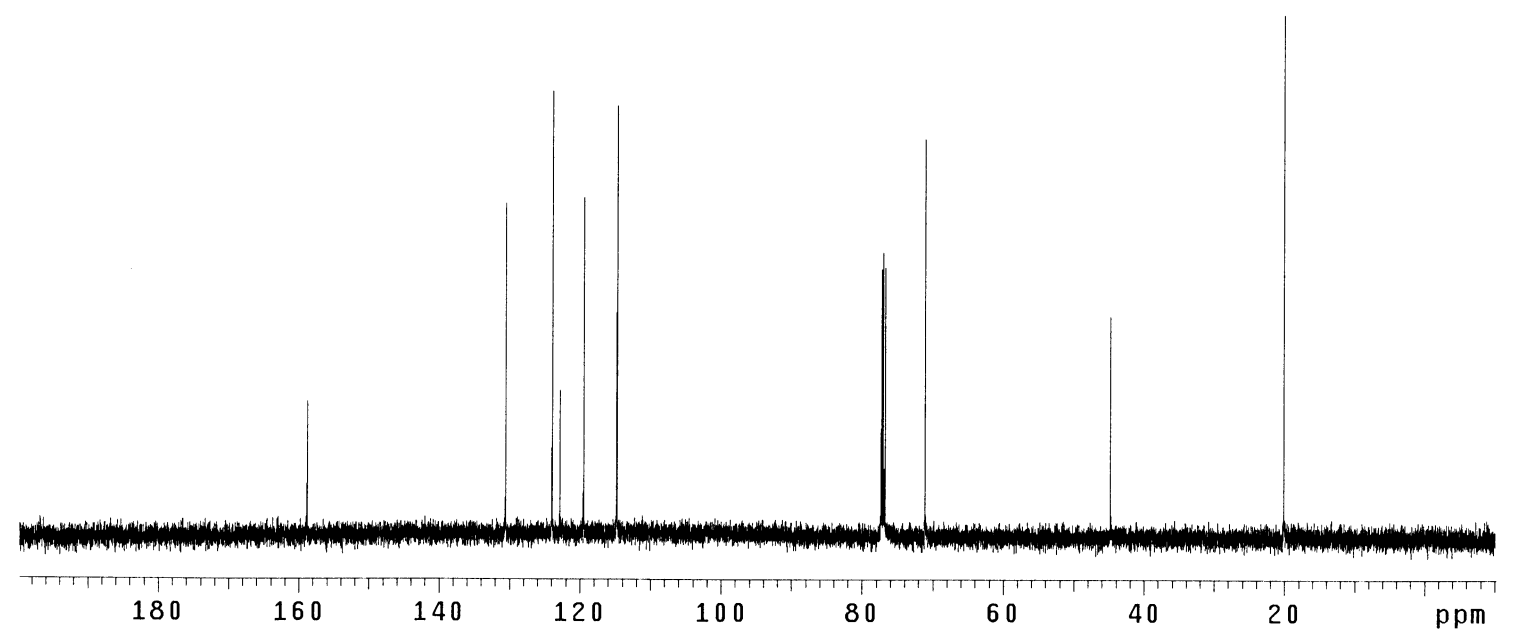




\section{${ }^{1} \mathrm{H}$ NMR $\left(500 \mathrm{MHz}, \mathrm{CDCl}_{3}\right)$ of $\mathbf{3 c}$}
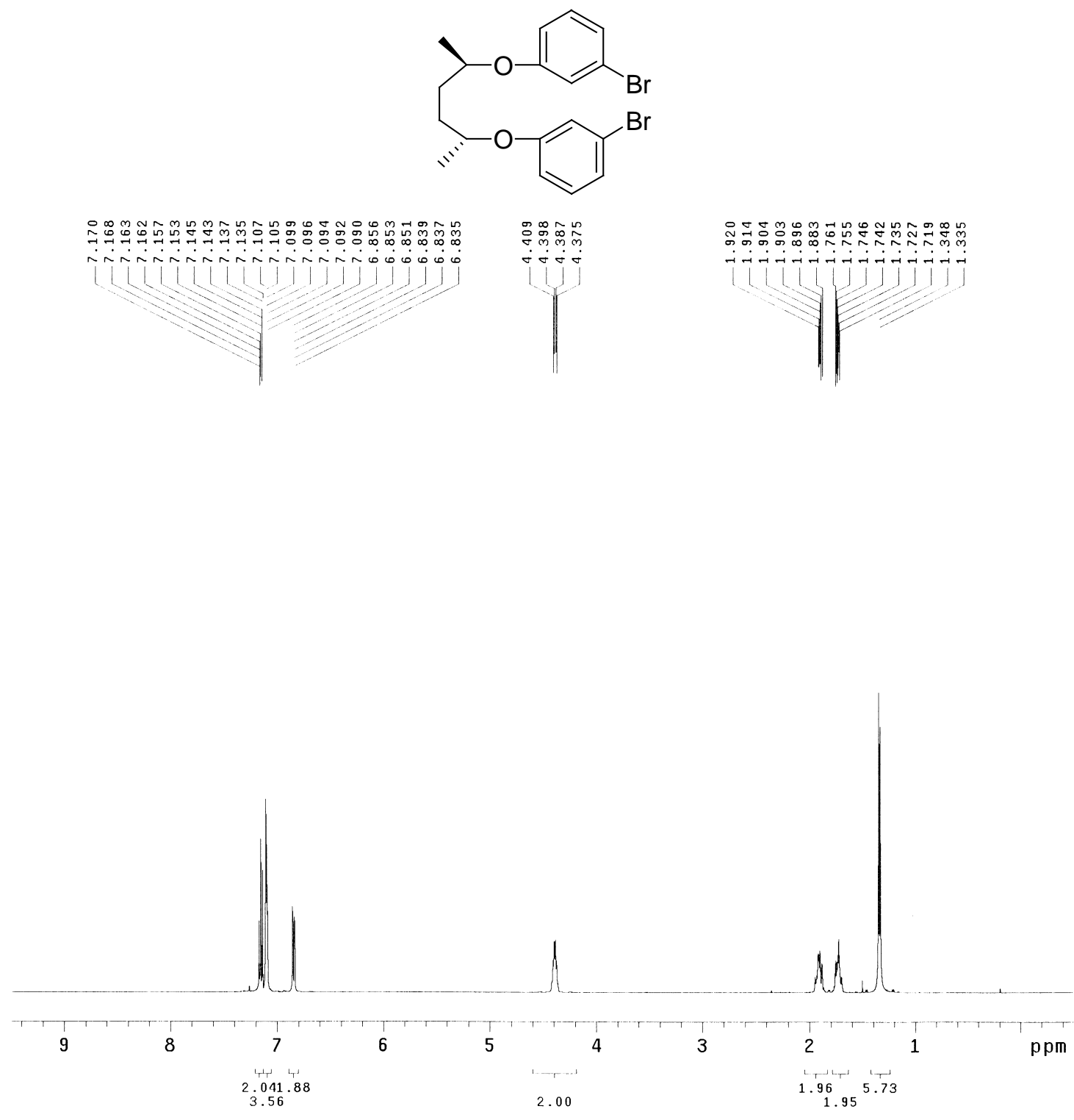
${ }^{13} \mathrm{C}$ NMR $\left(125 \mathrm{MHz}, \mathrm{CDCl}_{3}\right)$ of $\mathbf{3 c}$<smiles></smiles>
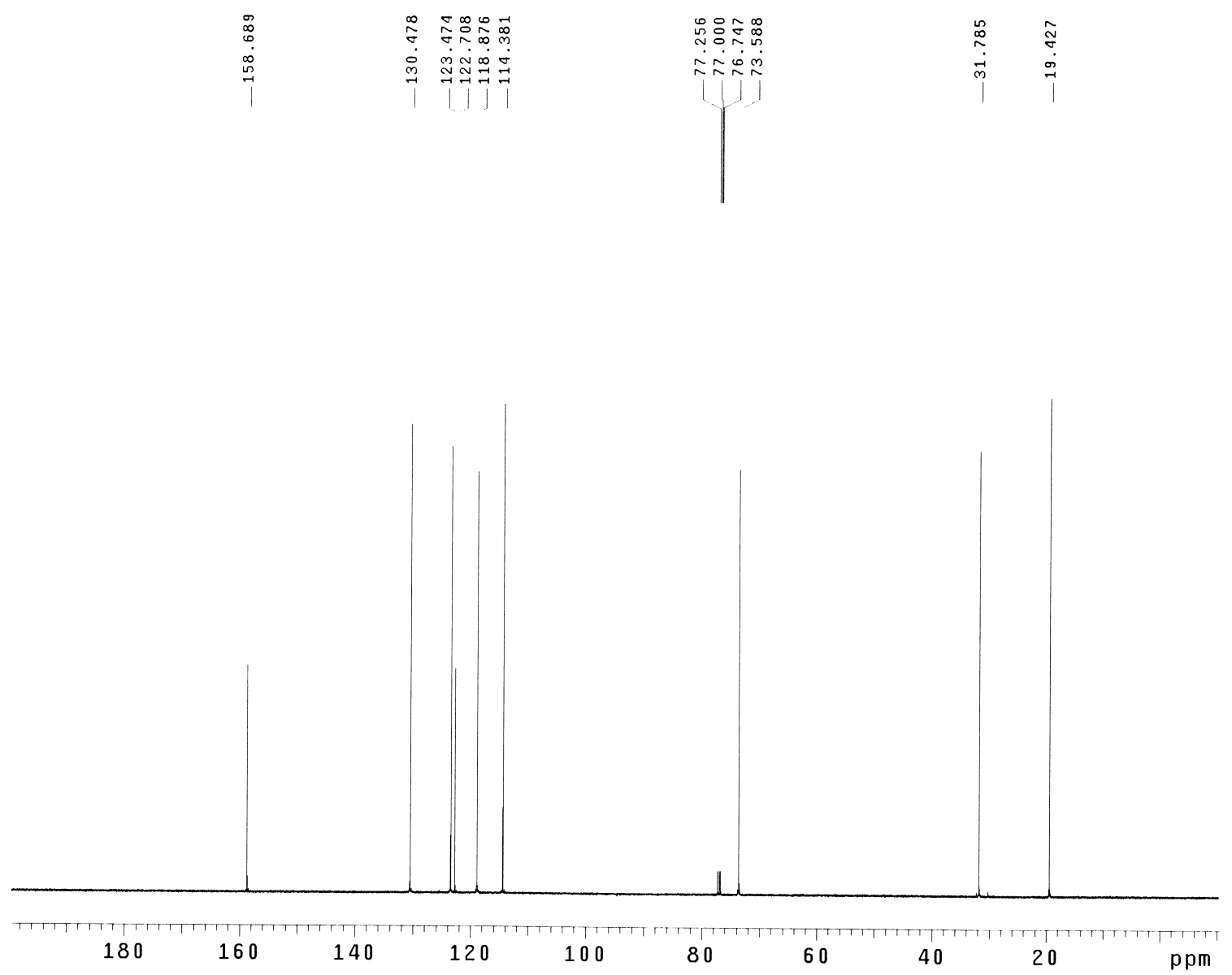


\section{${ }^{1} \mathrm{H}$ NMR $\left(500 \mathrm{MHz}, \mathrm{CDCl}_{3}\right)$ of $\mathbf{4 b}$}

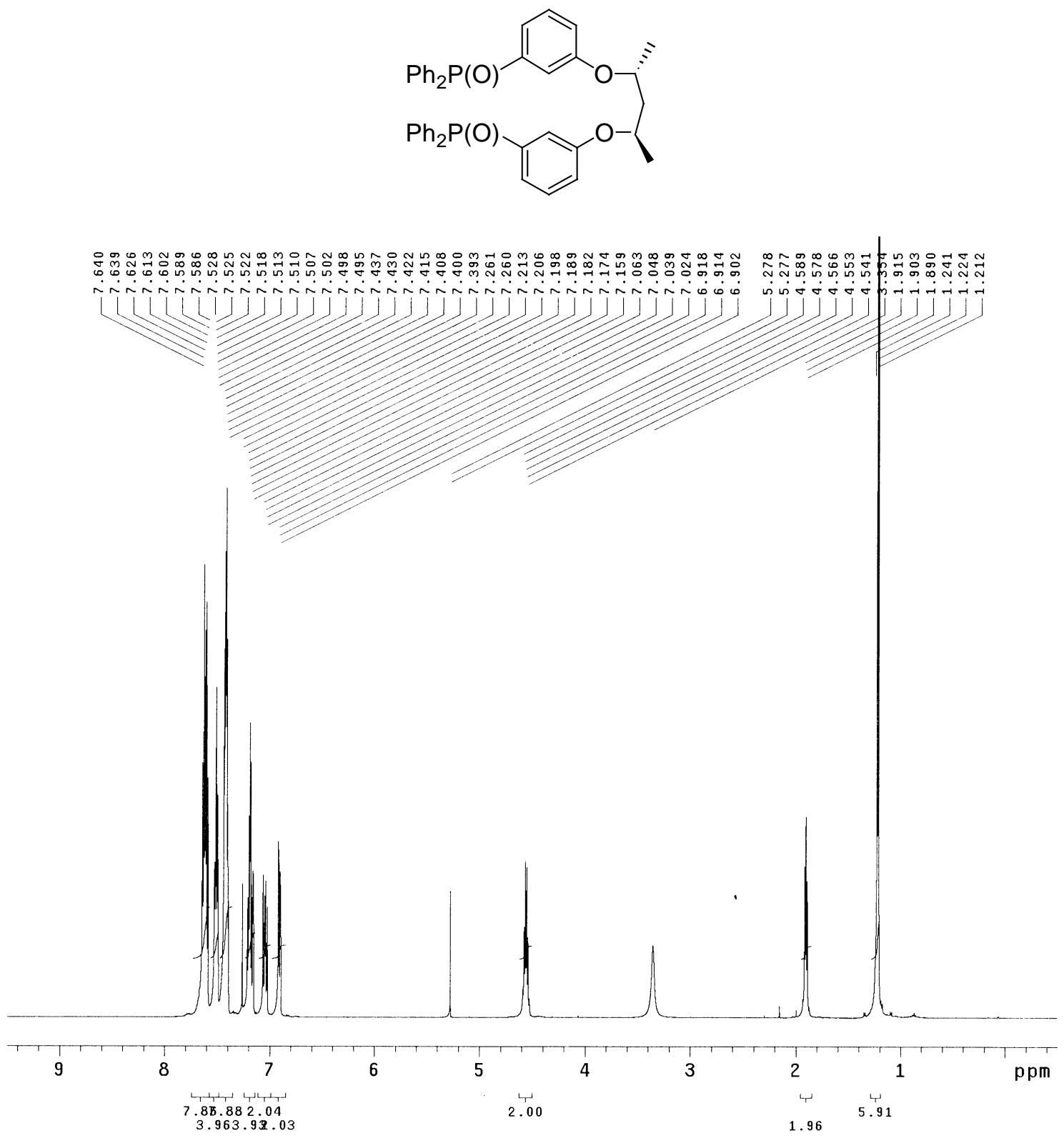


${ }^{31} \mathrm{P}$ NMR $\left(202 \mathrm{MHz}, \mathrm{CDCl}_{3}\right)$ of $\mathbf{4 b}$
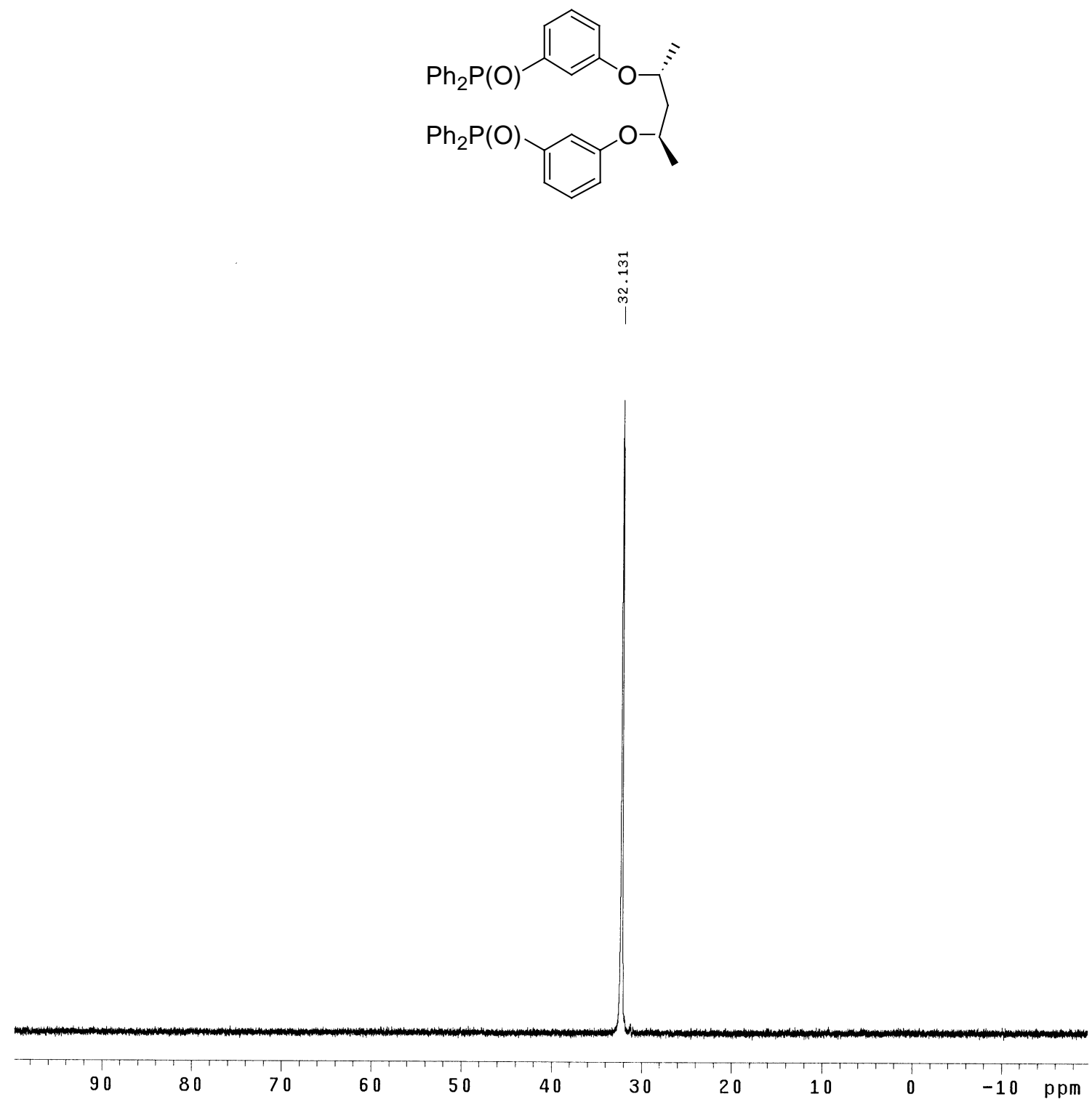
${ }^{13} \mathrm{C}$ NMR (125 MHz, $\left.\mathrm{CDCl}_{3}\right)$ of $\mathbf{4 b}$<smiles>CC(C)Oc1cccc(Oc2cccc(OPc3ccccc3)c2)c1</smiles>

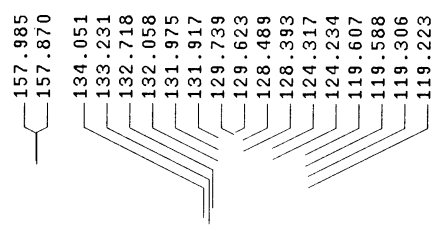

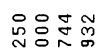

ㄷํ웅

(j)
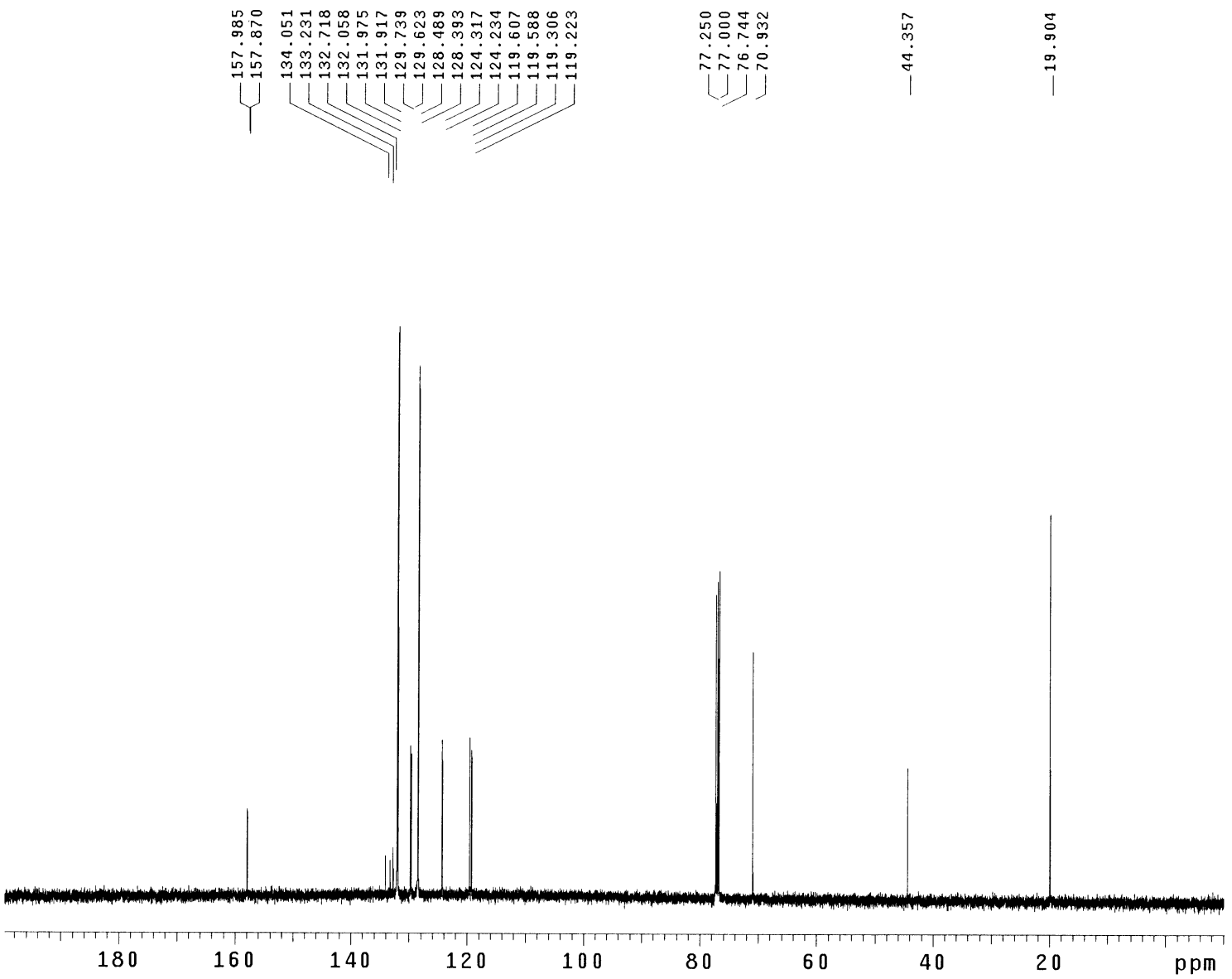


\section{${ }^{1} \mathrm{H}$ NMR $\left(500 \mathrm{MHz}, \mathrm{CDCl}_{3}\right)$ of $\mathbf{4 c}$}
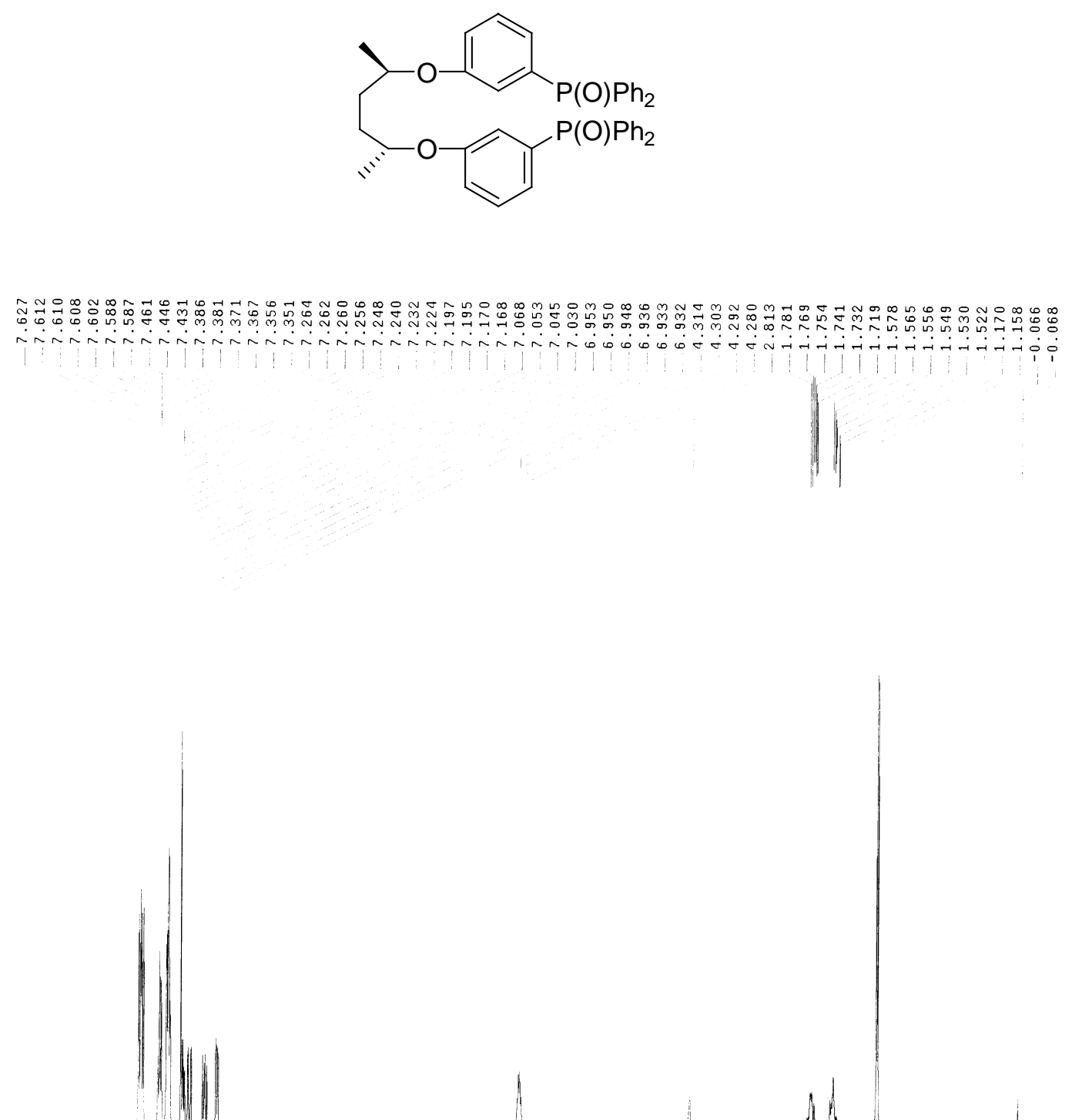

9

8

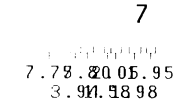

6

5

4

2.00
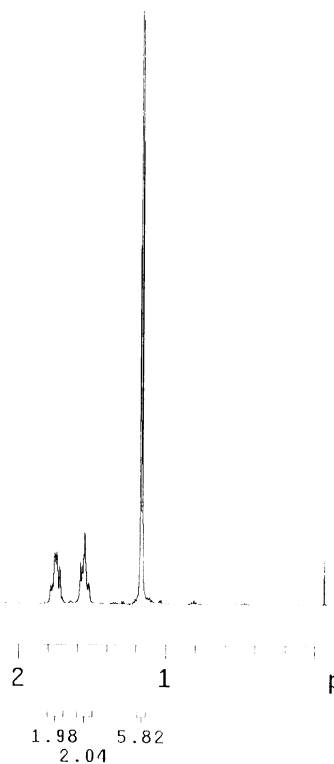
${ }^{31} \mathrm{P} \mathrm{NMR}\left(202 \mathrm{MHz}, \mathrm{CDCl}_{3}\right)$ of $\mathbf{4 c}$

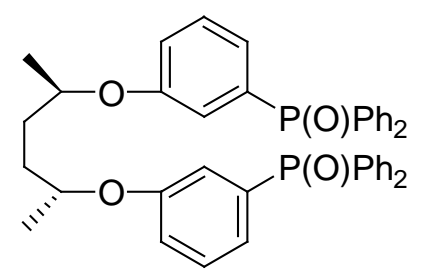

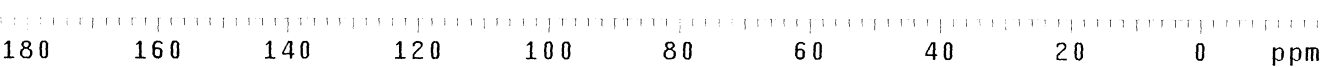

S-13 
${ }^{13} \mathrm{C}$ NMR $\left(125 \mathrm{MHz}, \mathrm{CDCl}_{3}\right)$ of $\mathbf{4 c}$
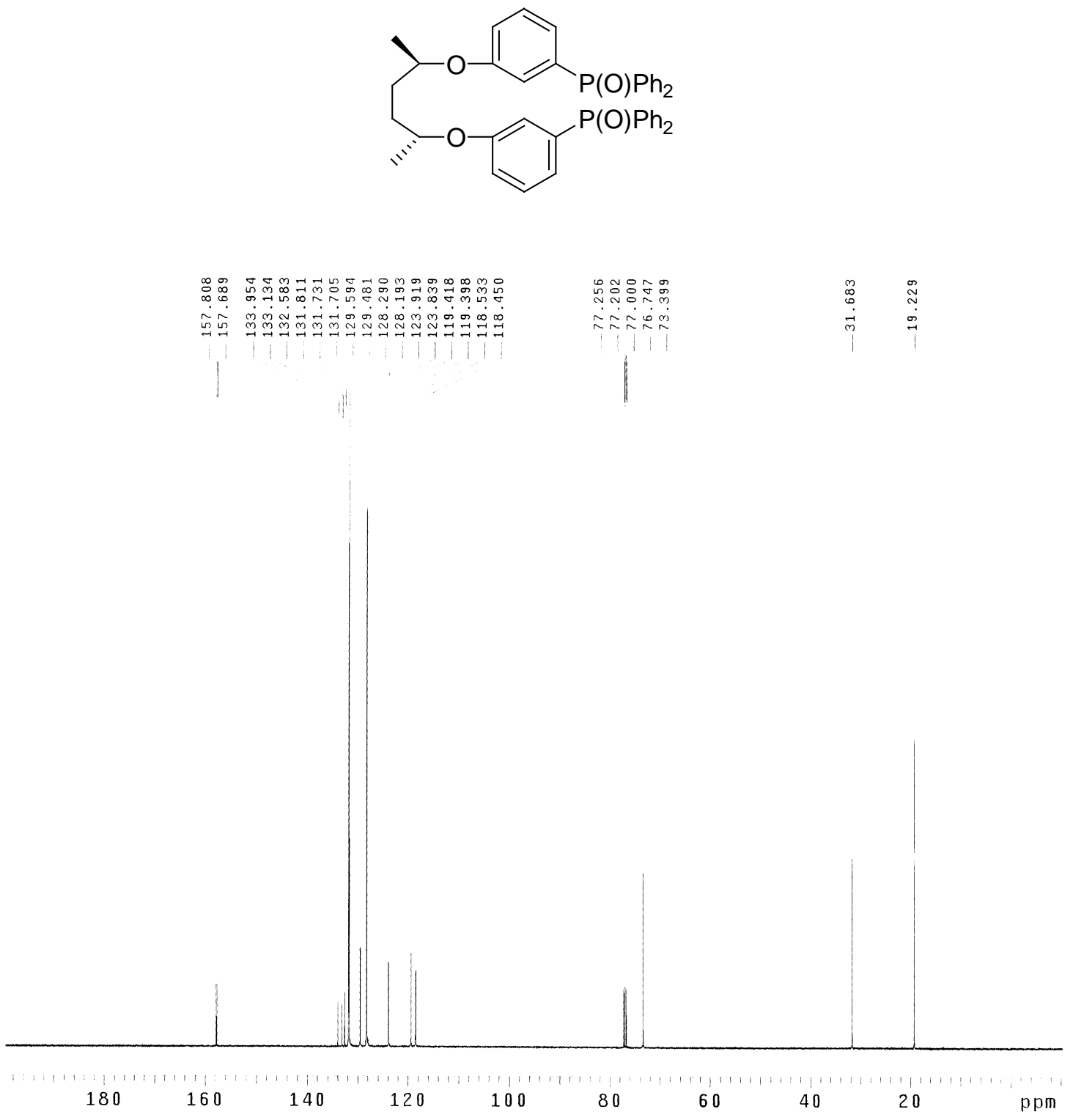


\section{${ }^{1} \mathrm{H}$ NMR $\left(500 \mathrm{MHz}, \mathrm{CDCl}_{3}\right)$ of $\mathbf{5 b}$}
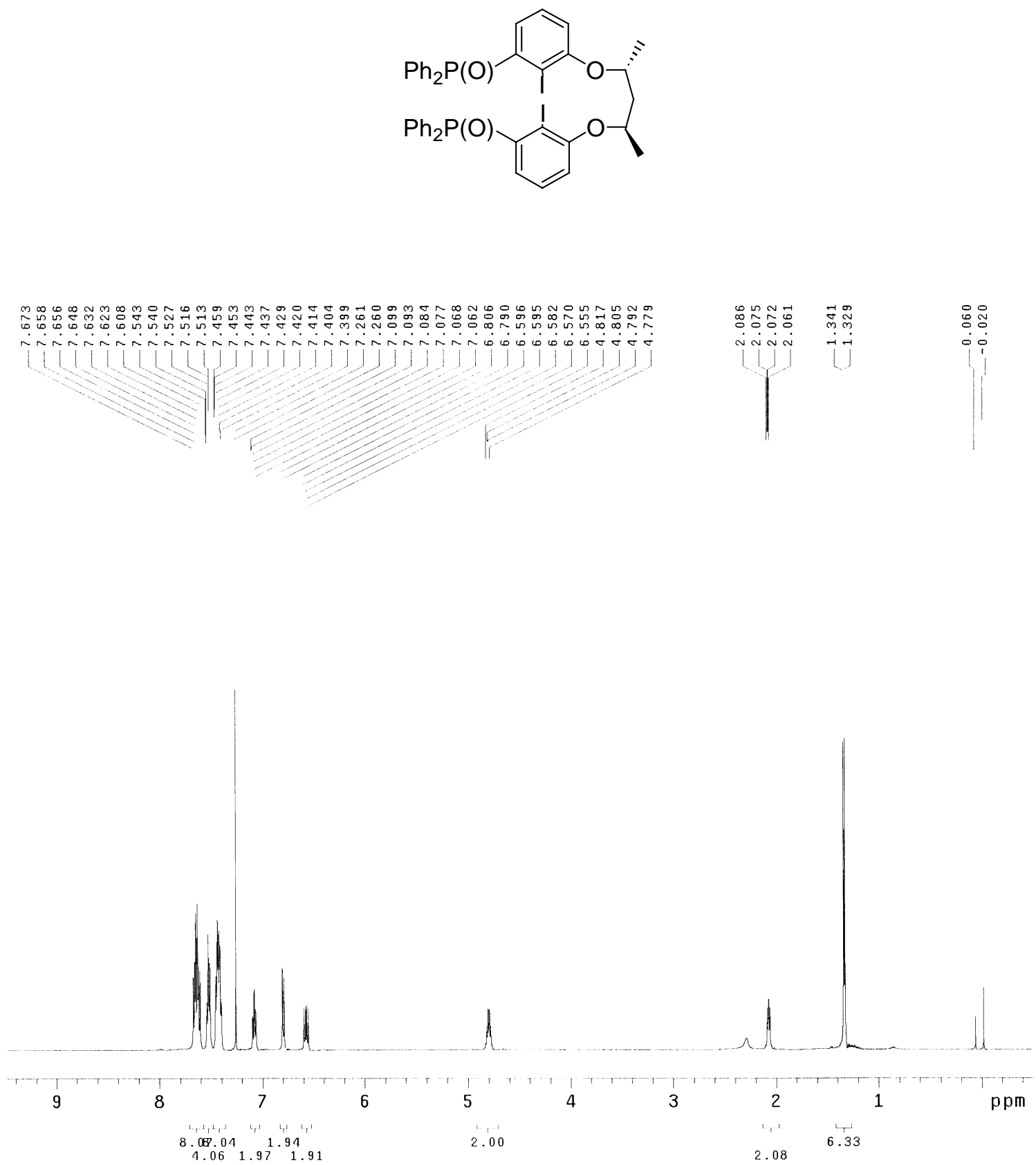
${ }^{31} \mathrm{P}$ NMR (202 MHz, $\mathrm{CDCl}_{3}$ ) of $\mathbf{5 b}$
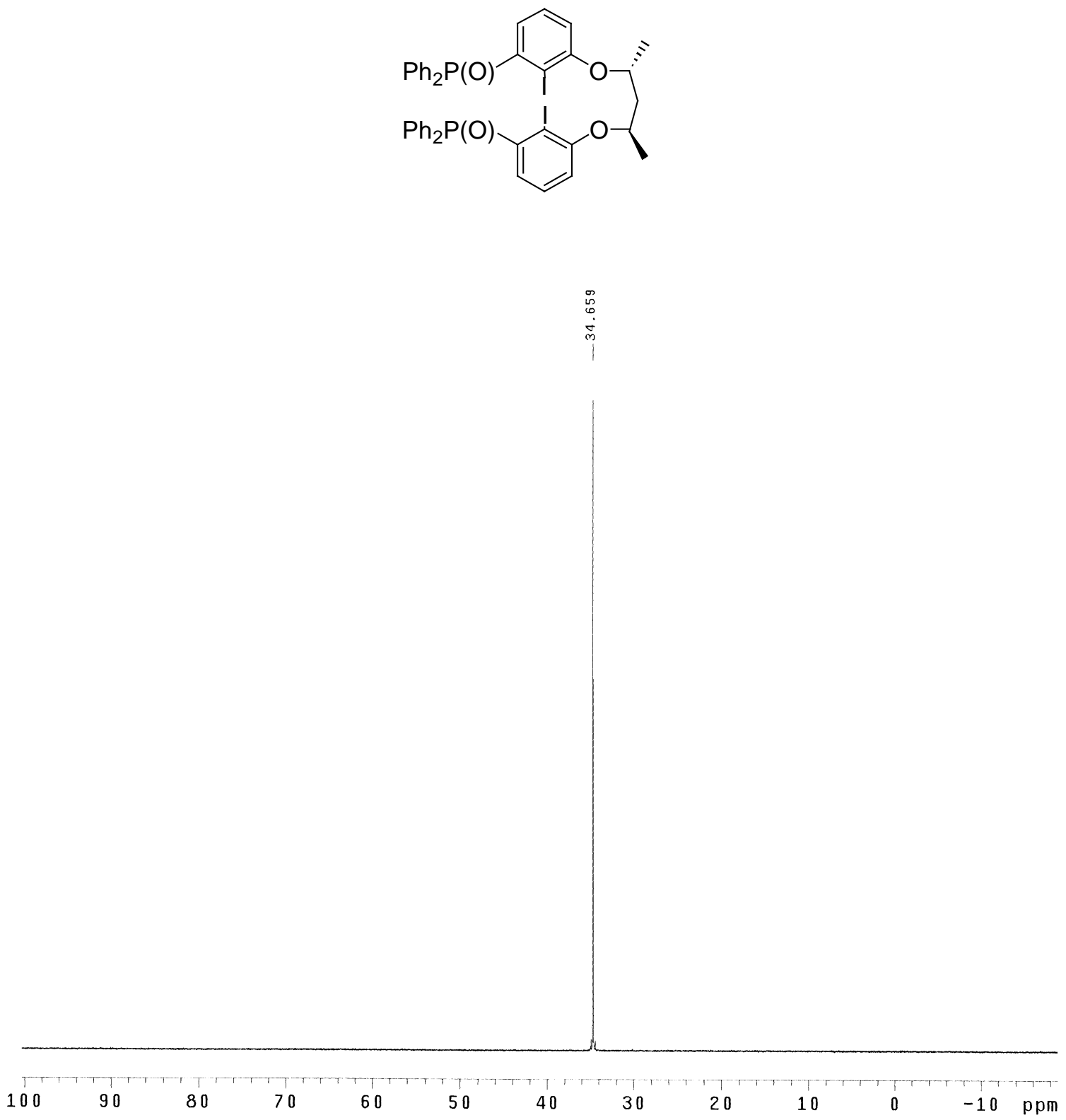

S-16 
${ }^{13} \mathrm{C}$ NMR $\left(125 \mathrm{MHz}, \mathrm{CDCl}_{3}\right)$ of $\mathbf{5 b}$
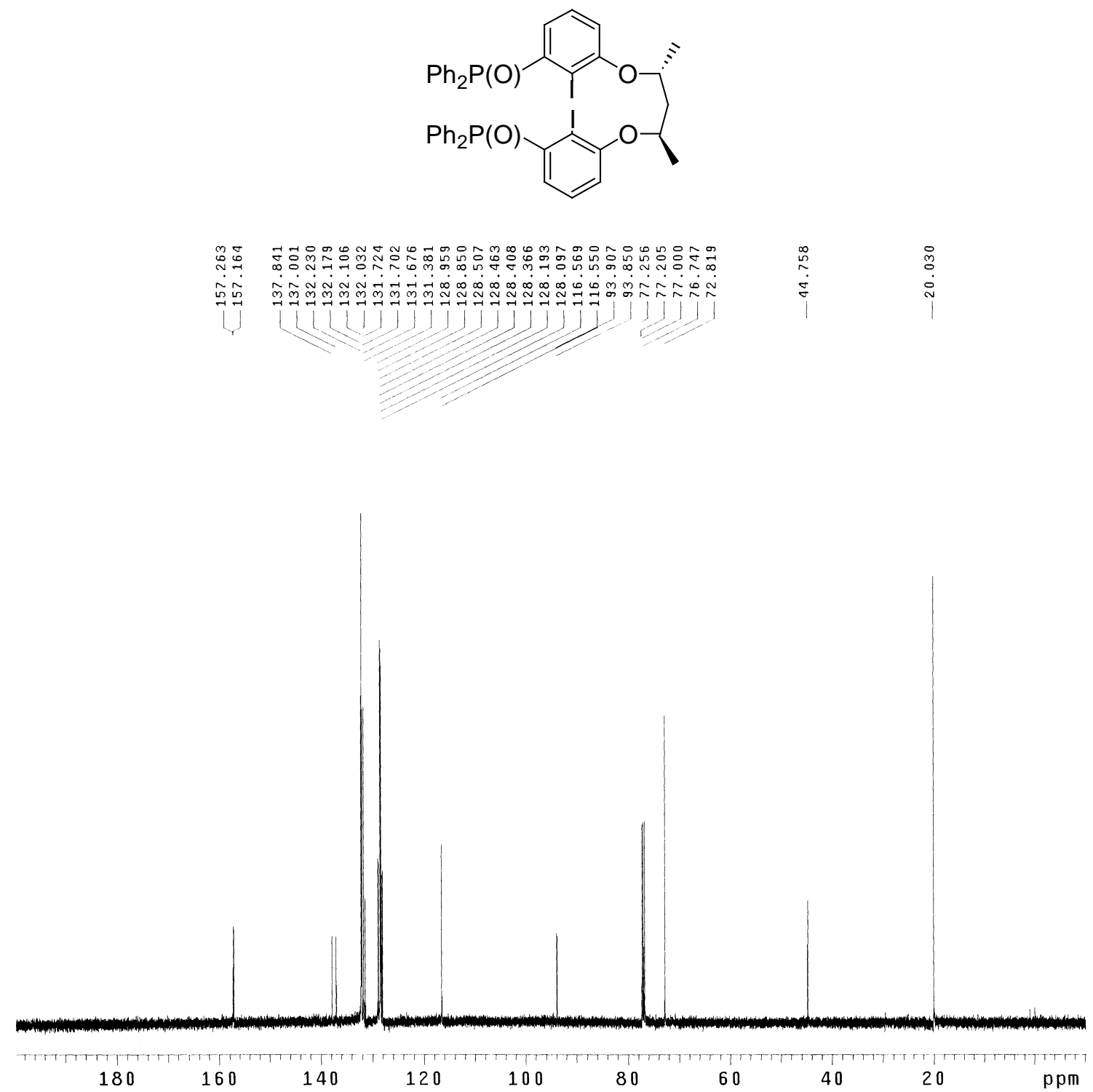


\section{${ }^{1} \mathrm{H}$ NMR $\left(500 \mathrm{MHz}, \mathrm{CDCl}_{3}\right)$ of $\mathbf{5 c}$}
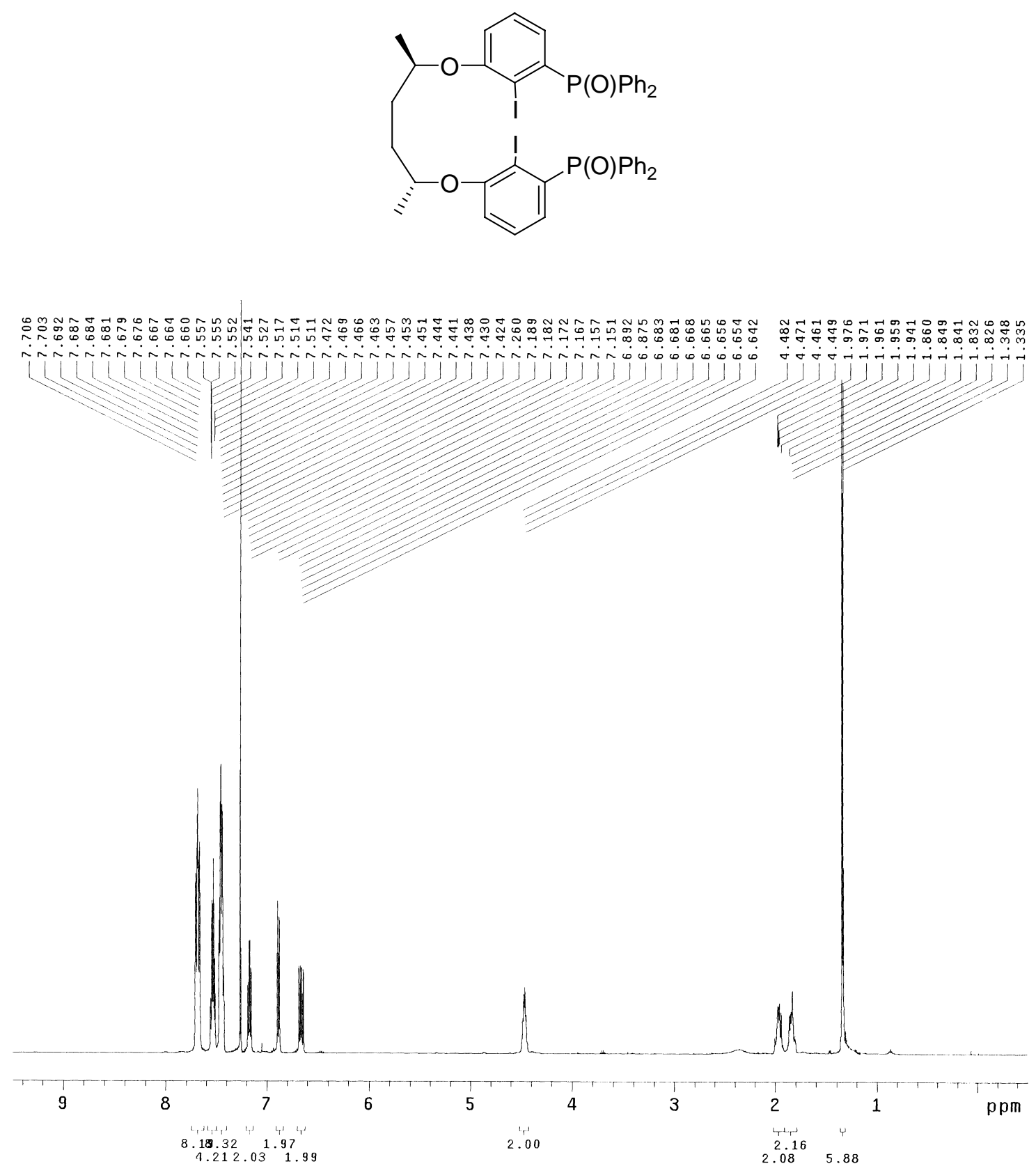
${ }^{31} \mathrm{P} \mathrm{NMR}\left(202 \mathrm{MHz}, \mathrm{CDCl}_{3}\right)$ of $\mathbf{5 c}$
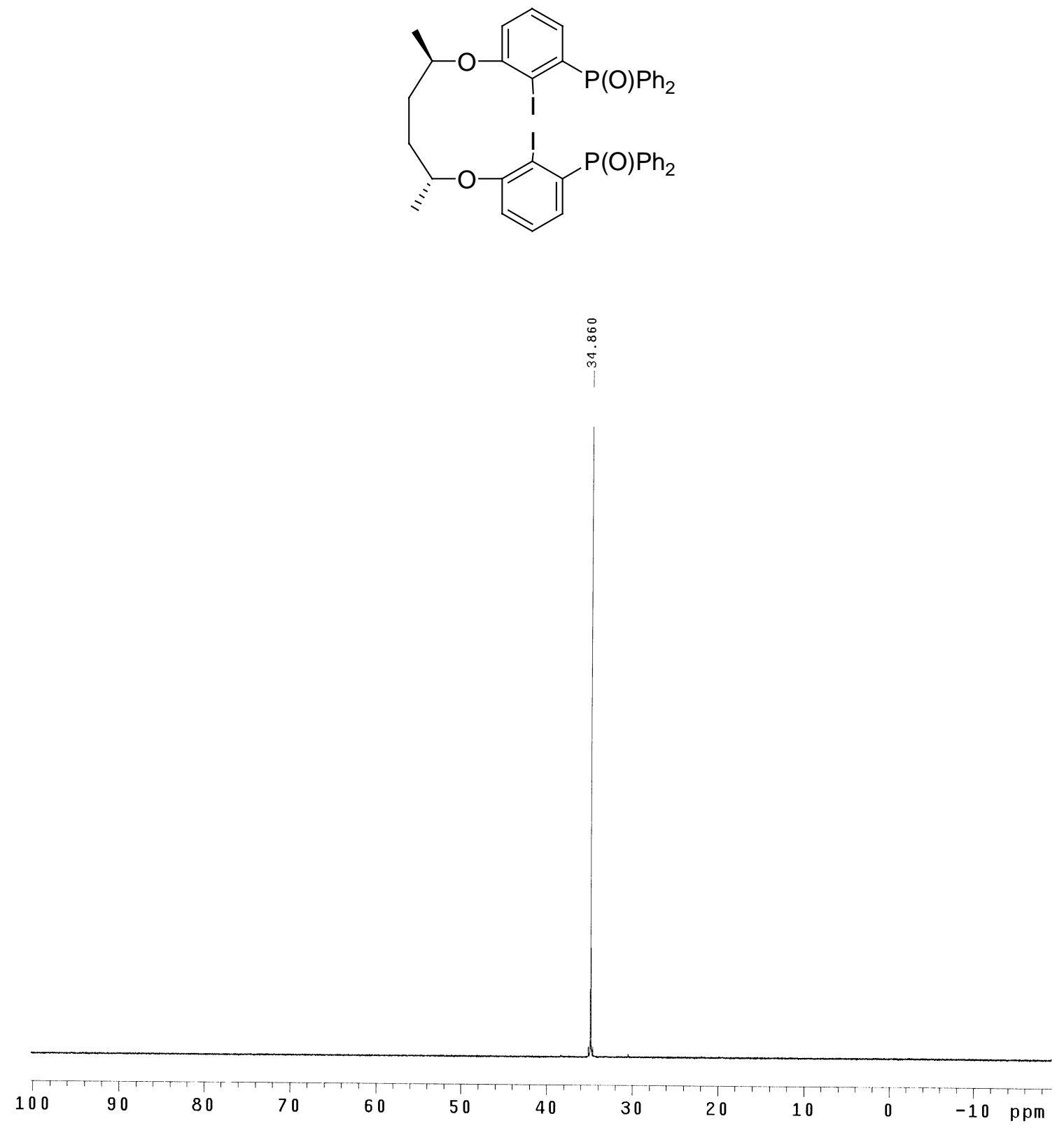
${ }^{13} \mathrm{C}$ NMR $\left(125 \mathrm{MHz}, \mathrm{CDCl}_{3}\right)$ of $\mathbf{5 c}$
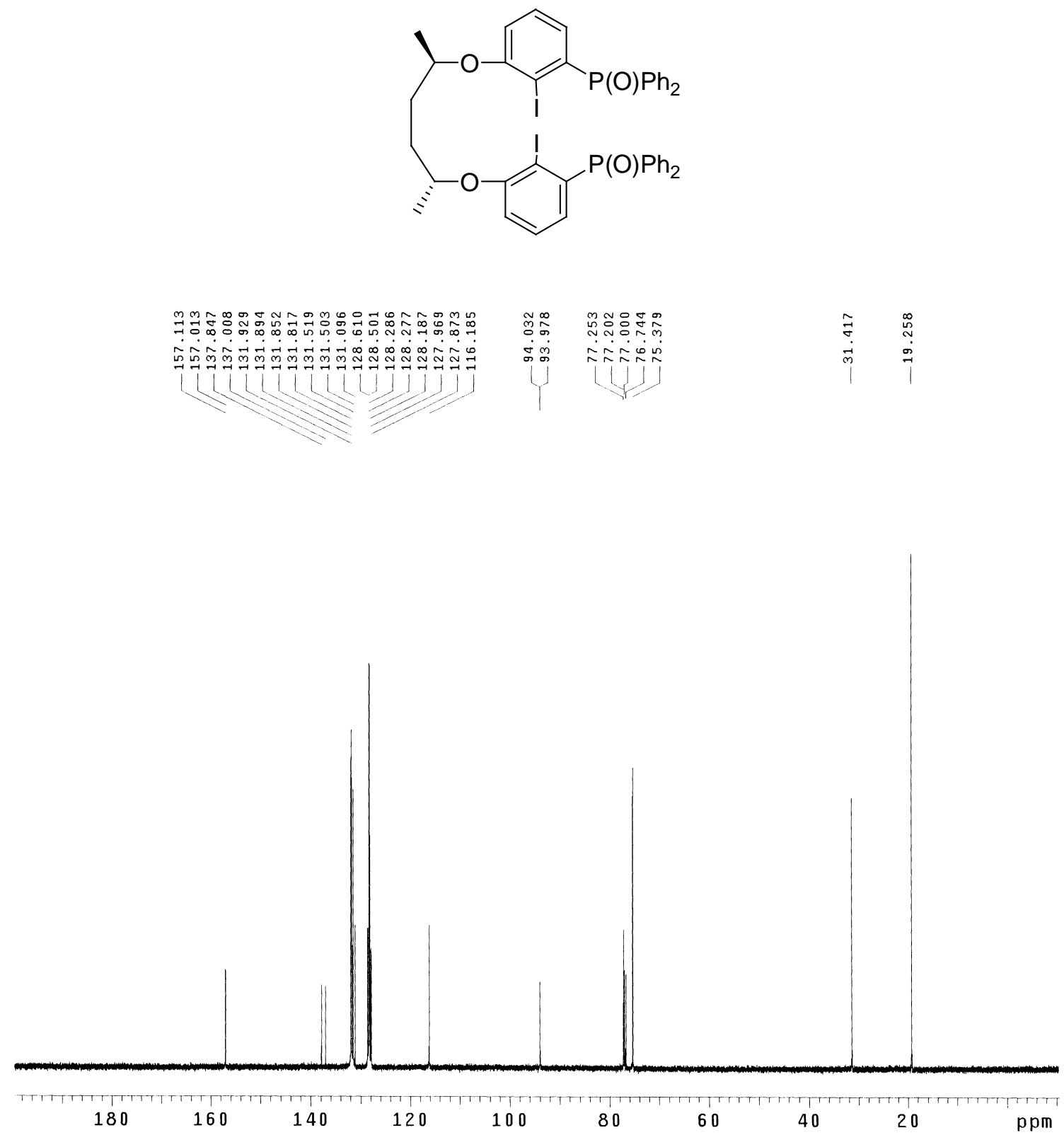


\section{${ }^{1} \mathrm{H}$ NMR $\left(500 \mathrm{MHz}, \mathrm{CDCl}_{3}\right)$ of $(S S S)-\mathbf{6 a}$}
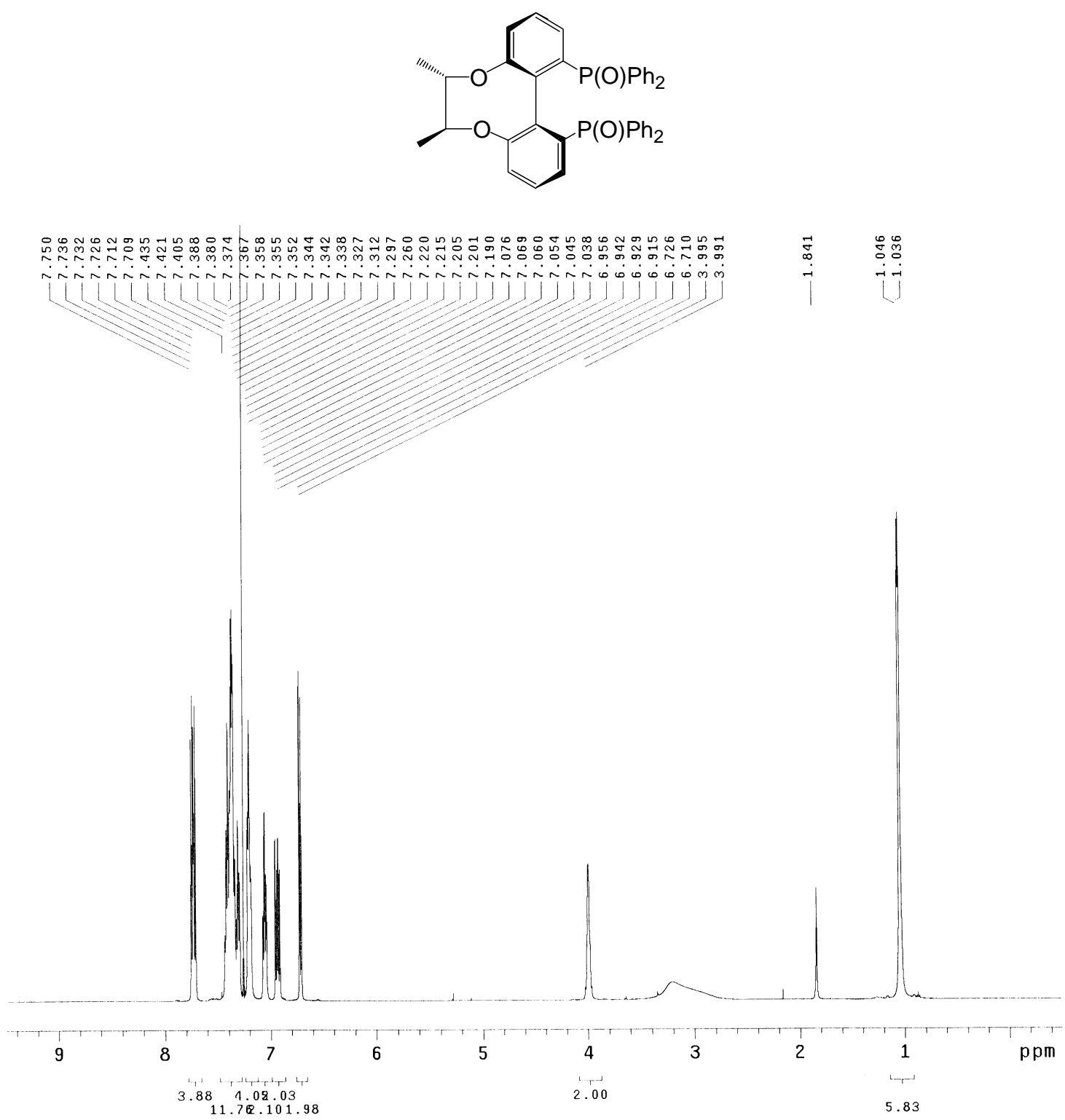
${ }^{31} \mathrm{P}$ NMR (202 MHz, $\left.\mathrm{CDCl}_{3}\right)$ of (SSS)-6a<smiles>CC1Oc2cccc(POc3ccccc3)c2-c2c(cccc2POc2ccccc2)O[C@H]1C</smiles>

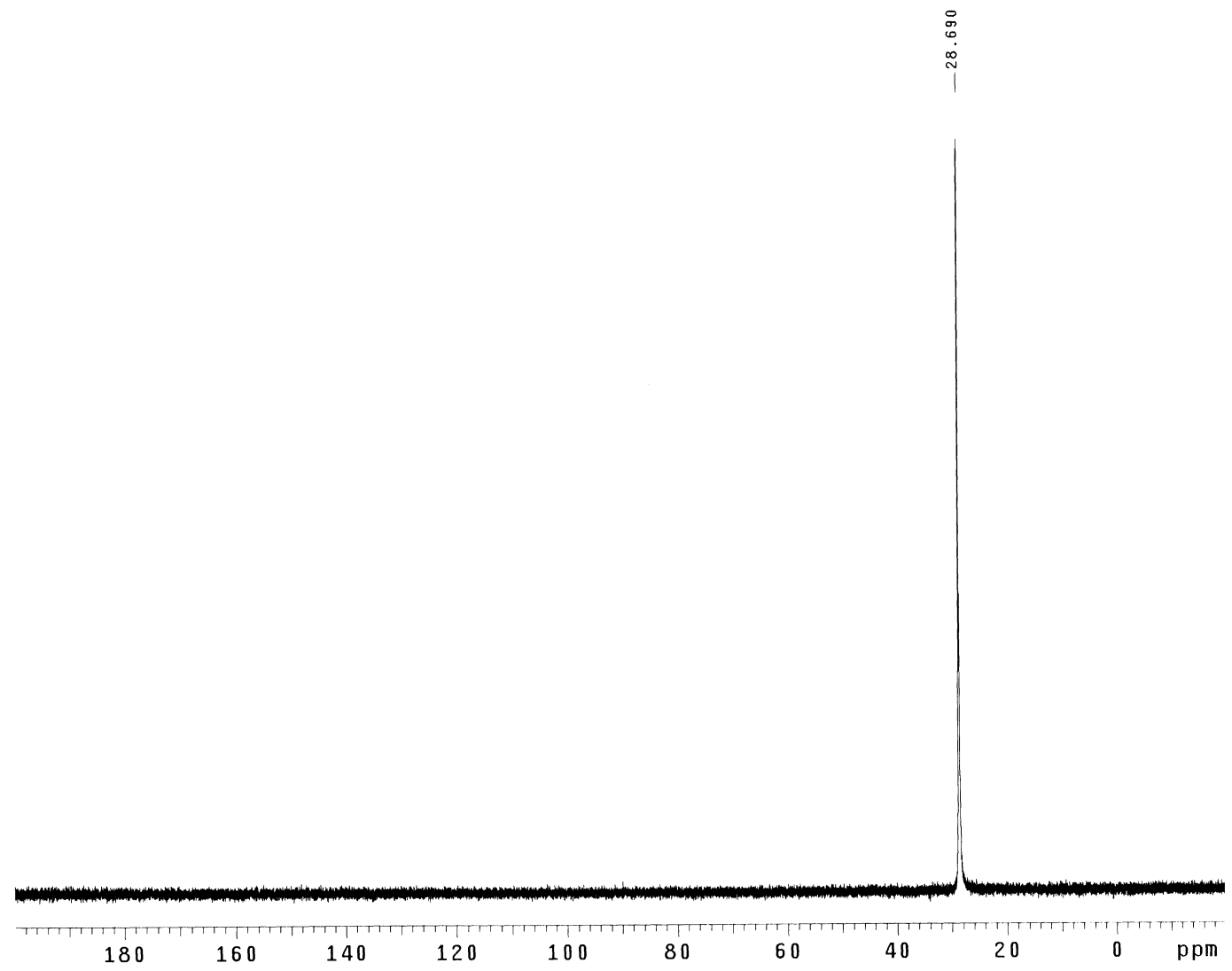


${ }^{13} \mathrm{C}$ NMR (125 MHz, $\left.\mathrm{CDCl}_{3}\right)$ of (SSS)-6a

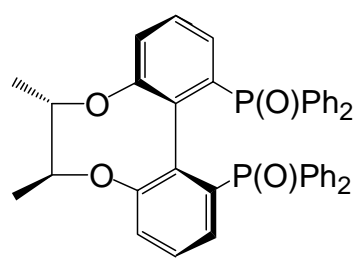

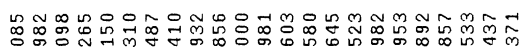

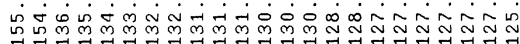

预总品导

$\stackrel{\cos 1}{\infty} \pi$

(1)

ஜூ

$1 \frac{x}{1}$

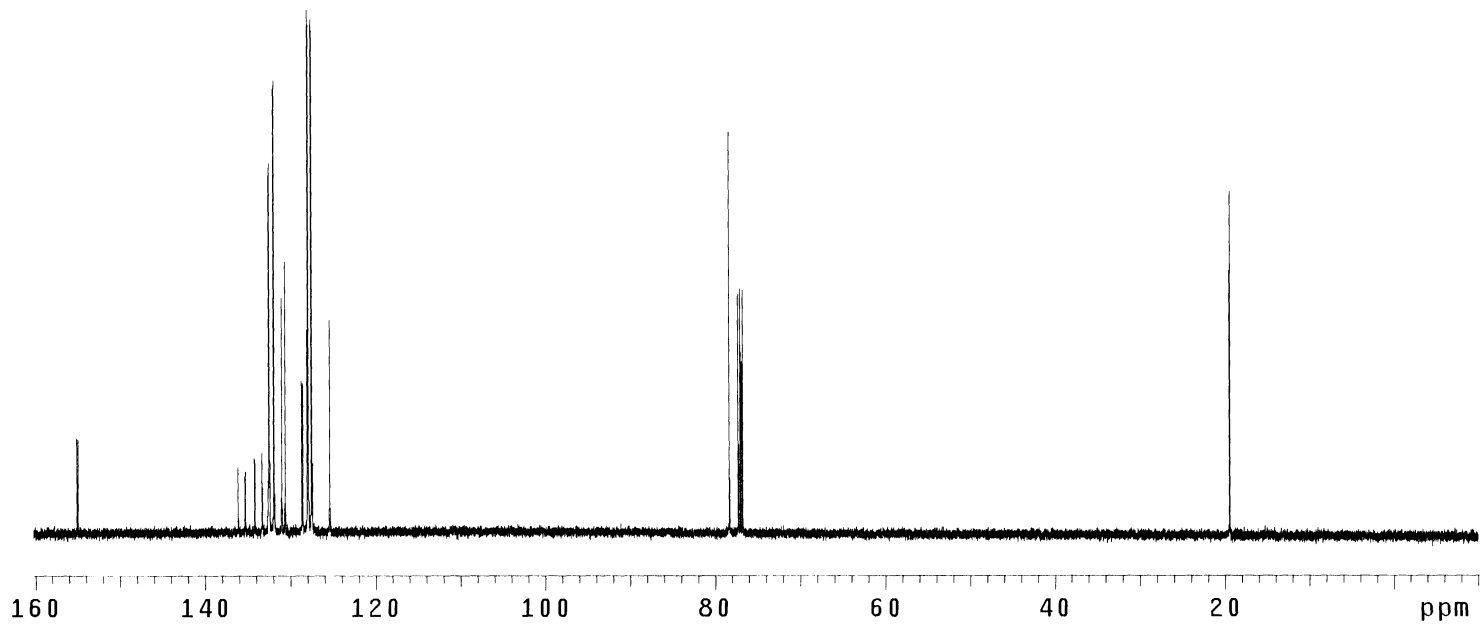


${ }^{1} \mathrm{H}$ NMR $\left(500 \mathrm{MHz}, \mathrm{CDCl}_{3}\right)$ of $($ SRR $)-\mathbf{6 b}$
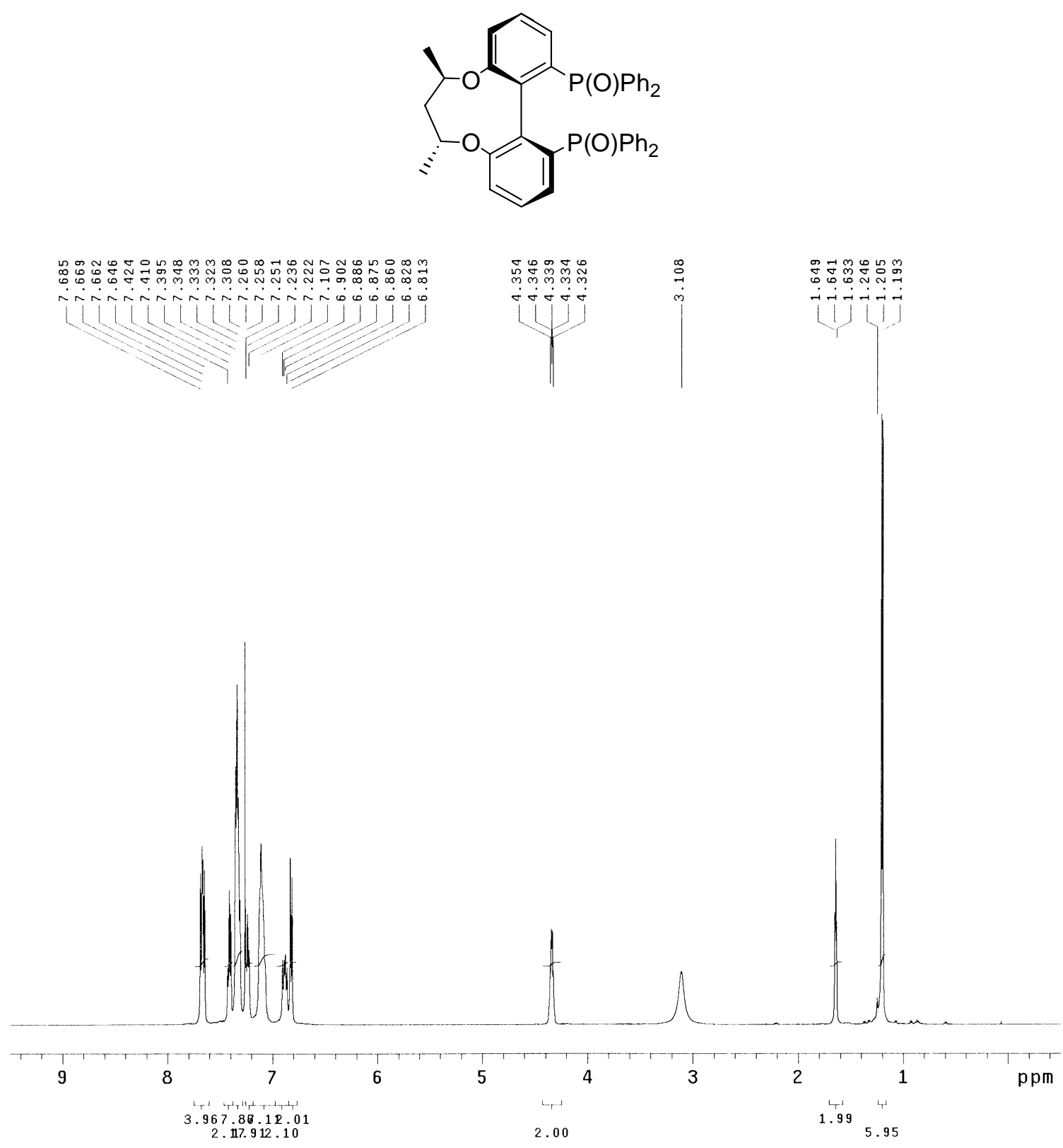
${ }^{31}$ P NMR (202 MHz, $\left.\mathrm{CDCl}_{3}\right)$ of (SRR)-6b
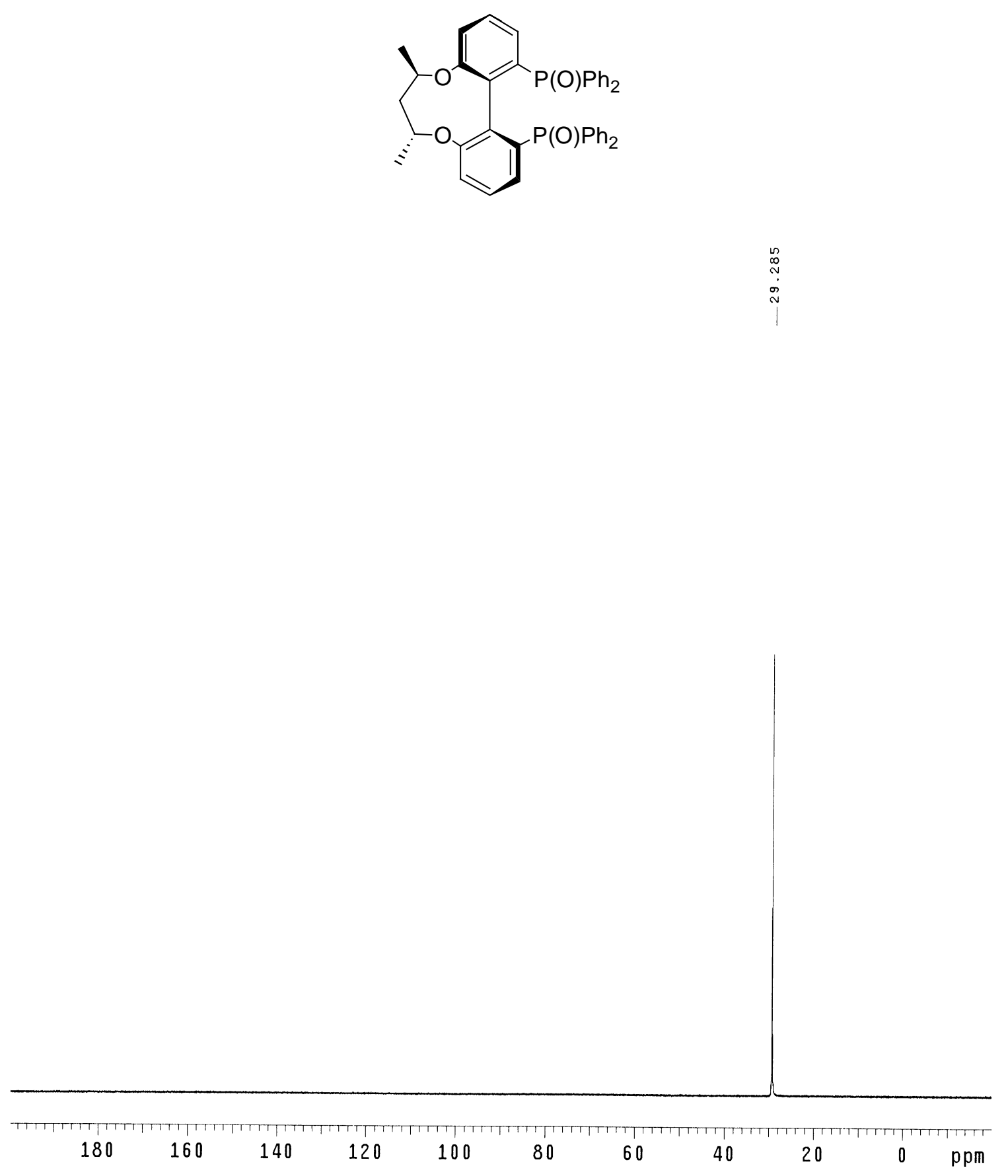
${ }^{13} \mathrm{C}$ NMR (125 MHz, $\left.\mathrm{CDCl}_{3}\right)$ of $(S R R)-\mathbf{6 b}$
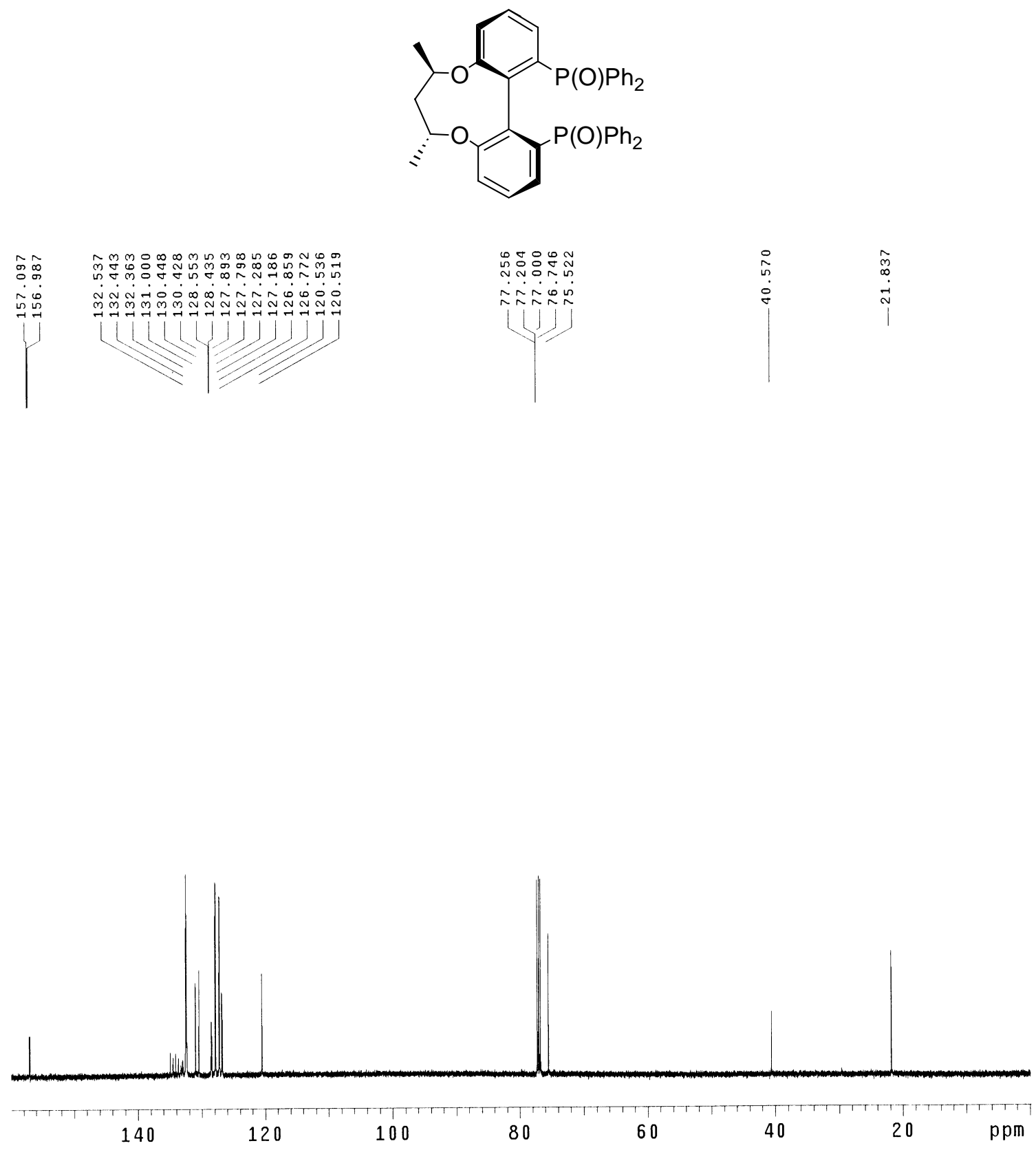
${ }^{1} \mathrm{H}$ NMR (500 MHz, $\left.\mathrm{CDCl}_{3}\right)$ of (SRR)-6c

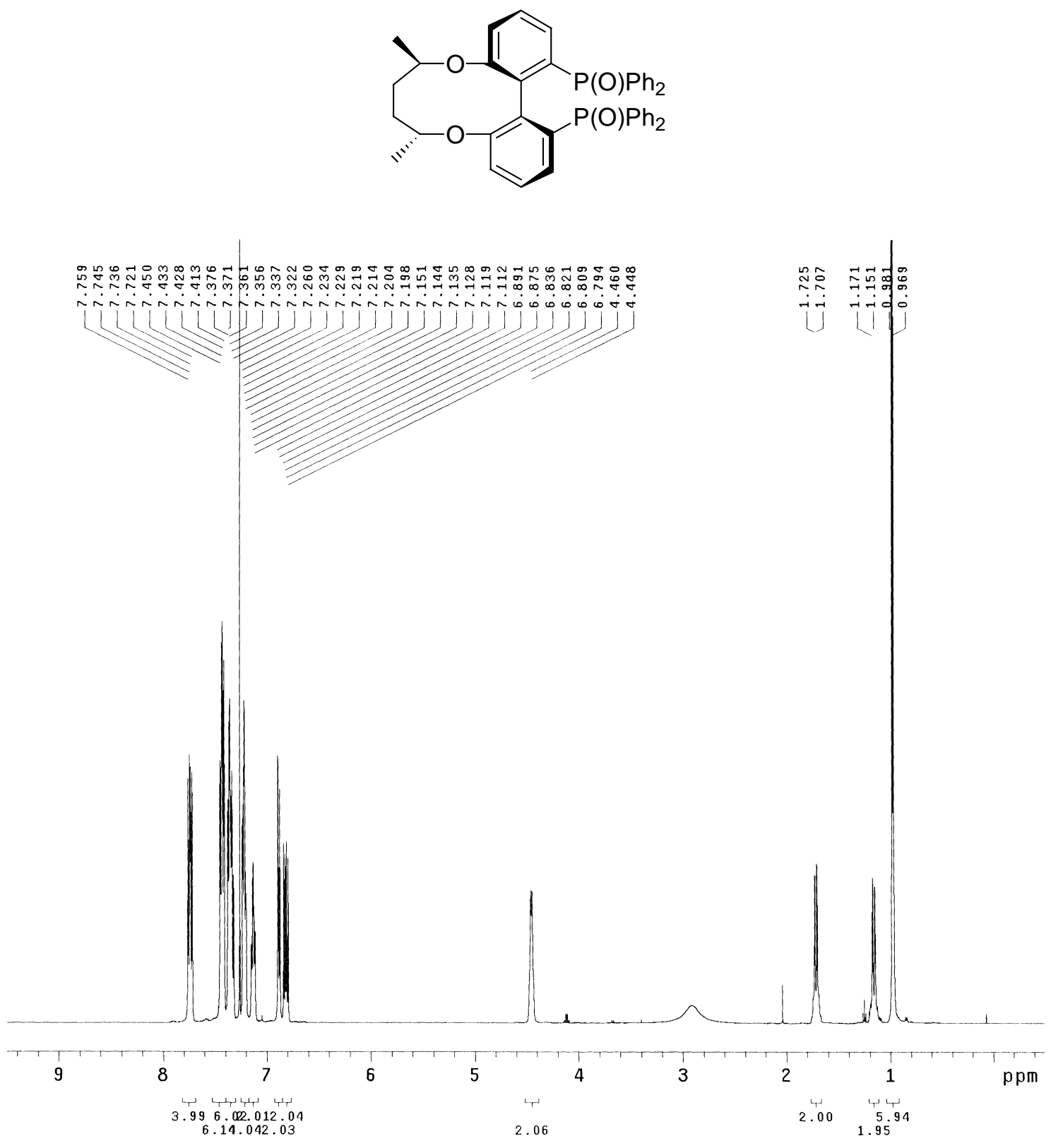


${ }^{31} \mathrm{P}$ NMR (202 MHz, $\left.\mathrm{CDCl}_{3}\right)$ of $(S R R)-\mathbf{6 c}$<smiles>C[C@@H]1CC[C@@H](C)Oc2cccc(POCc3ccccc3Pc3ccccc3)c2-c2c(P)cccc2O1</smiles>

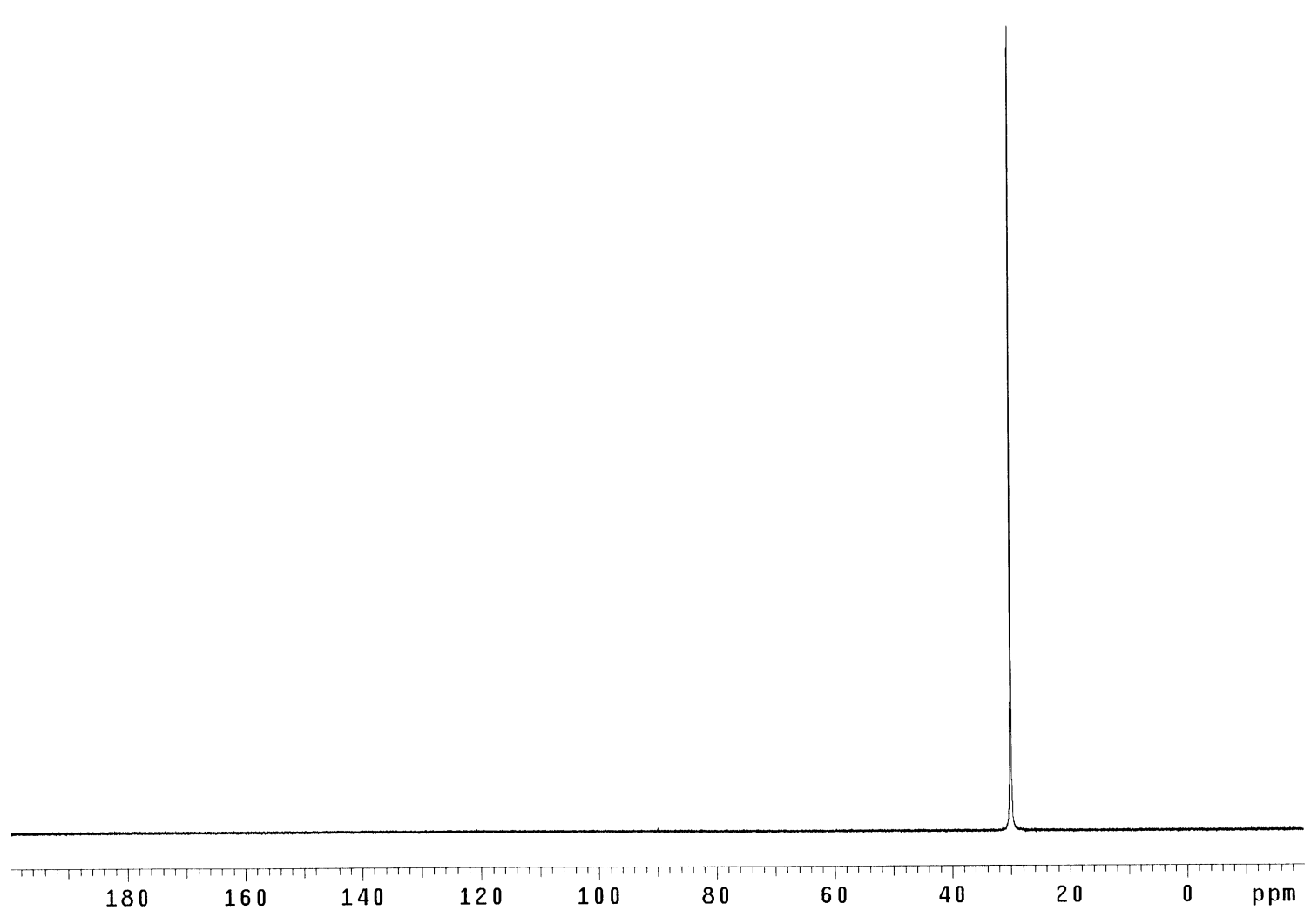


${ }^{13} \mathrm{C}$ NMR (125 MHz, $\left.\mathrm{CDCl}_{3}\right)$ of (SRR)-6c<smiles>C[C@@H]1CC[C@@H](C)Oc2cccc(POCCP)c2-c2c(P)cccc2O1</smiles>

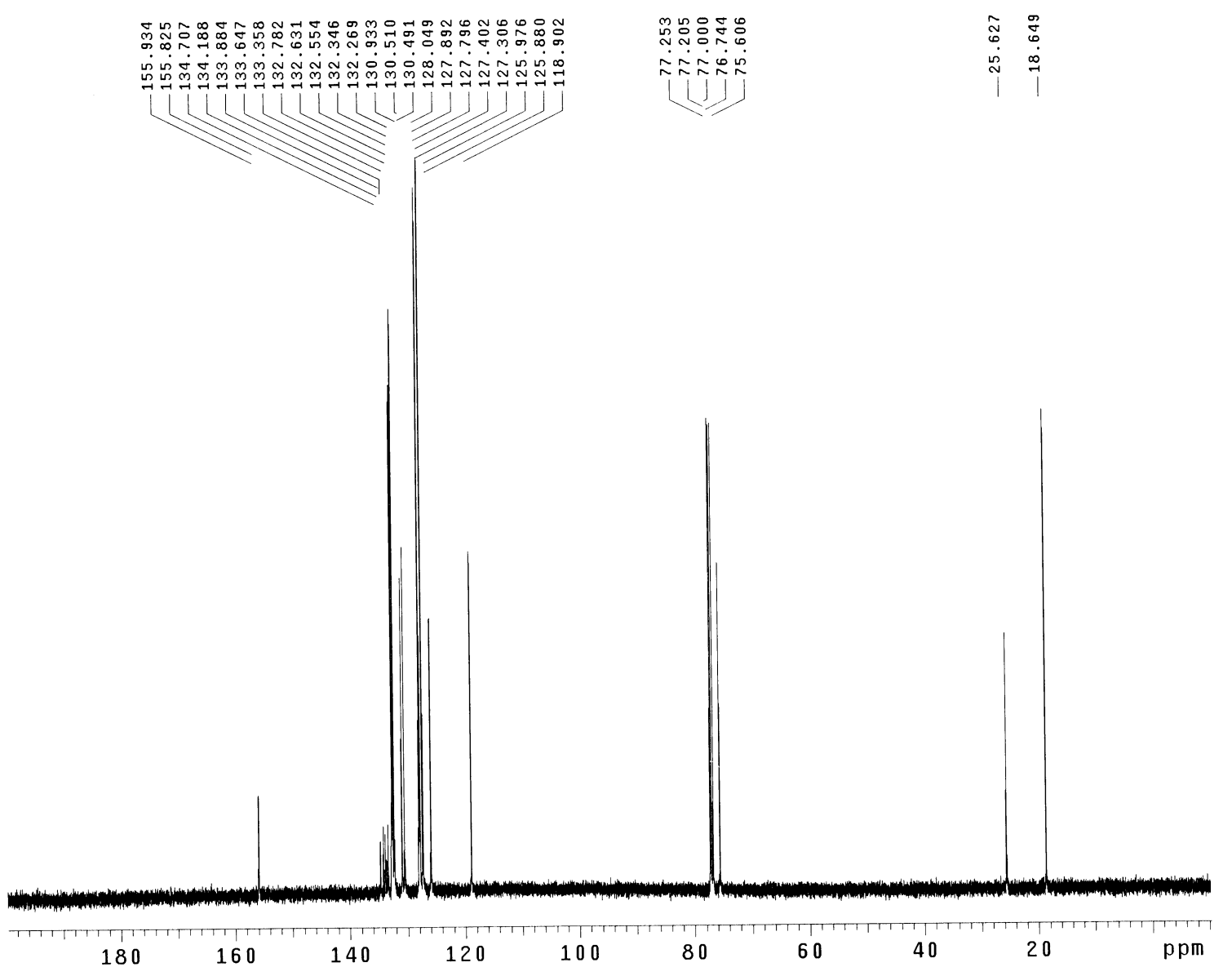


${ }^{1} \mathrm{H}$ NMR (500 MHz, CDCl $)$ of (SSS)-7a
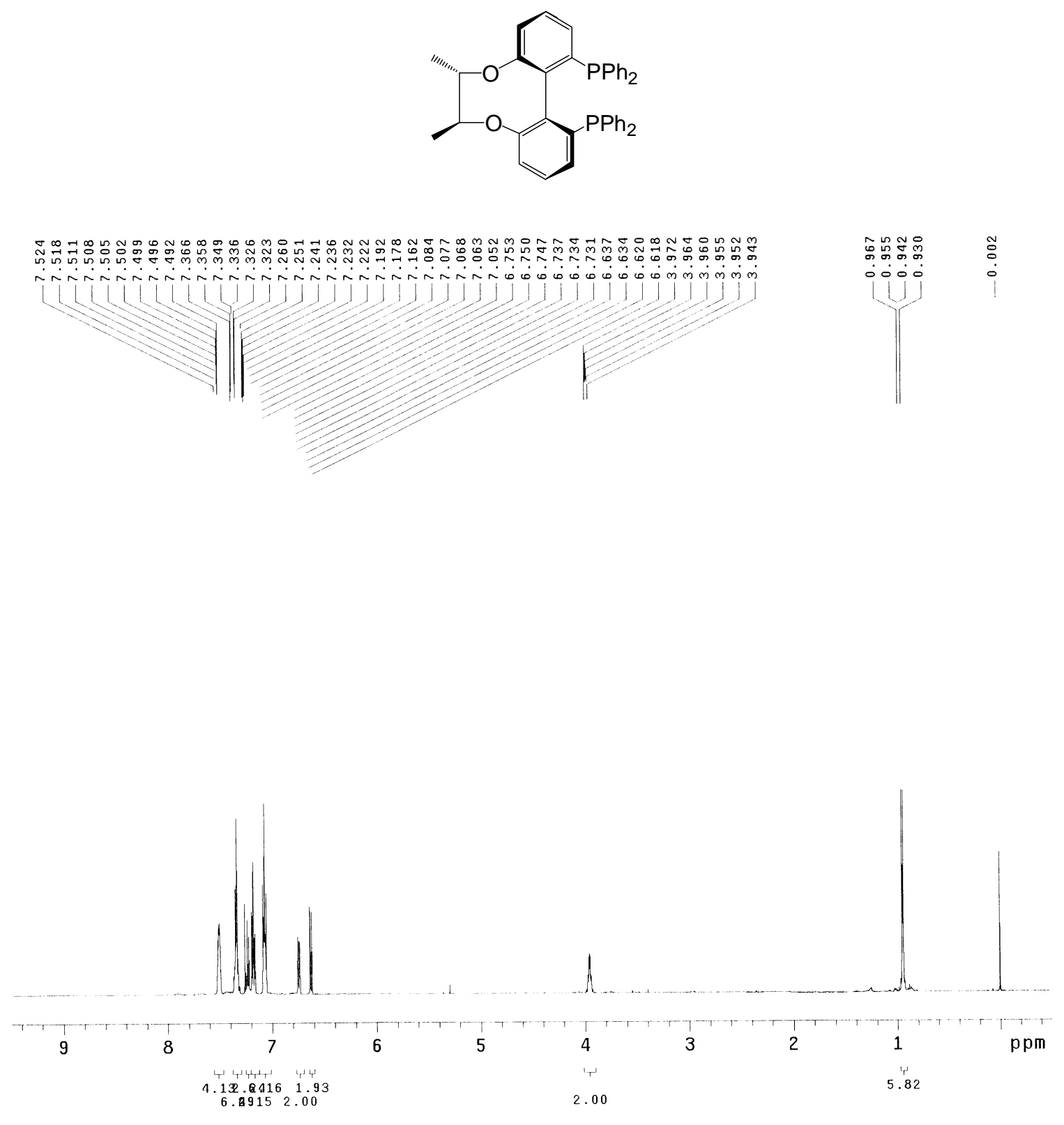
${ }^{31} \mathrm{P}$ NMR (202 MHz, $\left.\mathrm{CDCl}_{3}\right)$ of $(S S S)-7 \mathbf{a}$<smiles>CC1Oc2cccc(-c3ccccc3)c2-c2c(cccc2-c2ccccc2)O1</smiles>

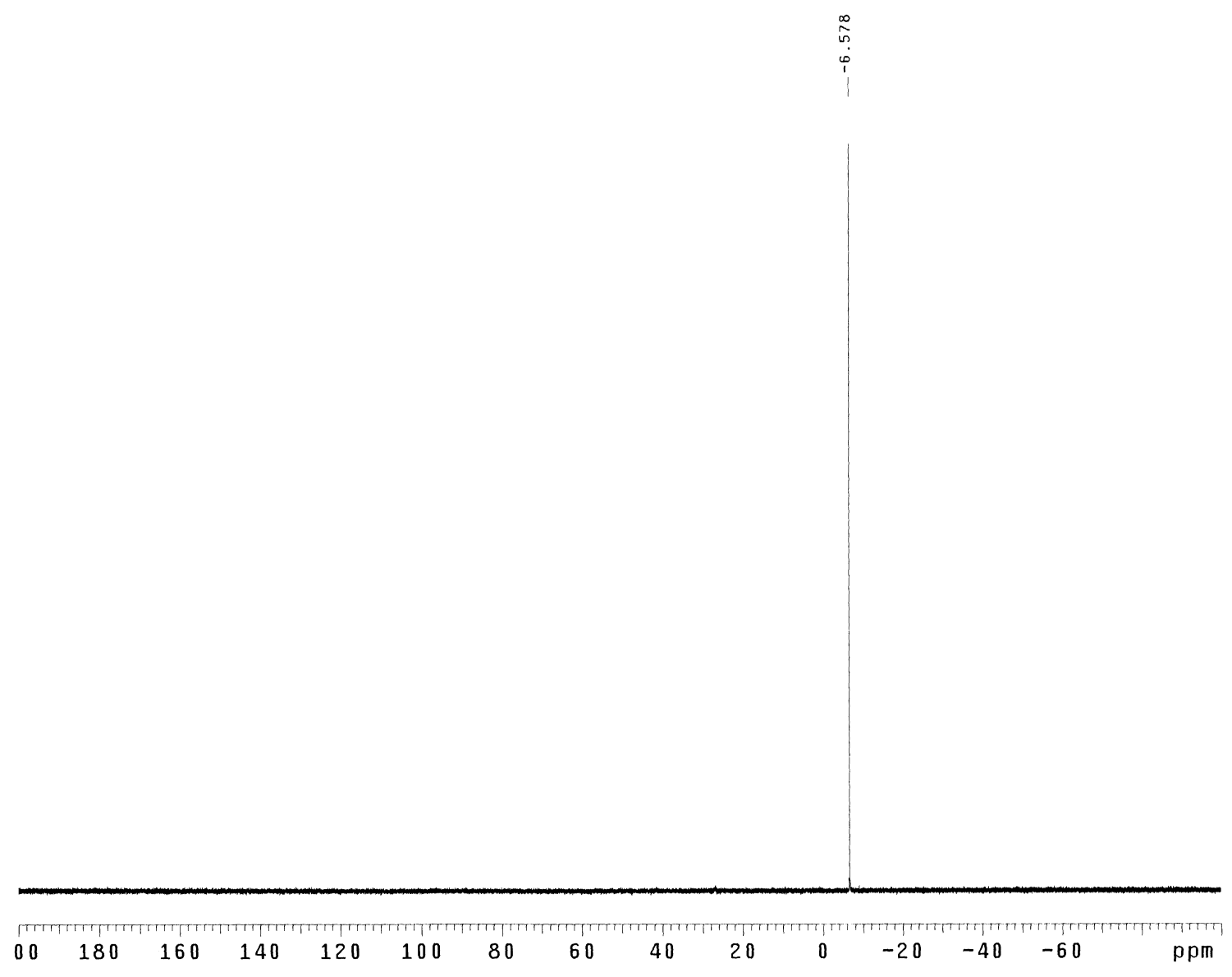


${ }^{13} \mathrm{C}$ NMR (125 MHz, $\left.\mathrm{CDCl}_{3}\right)$ of $(S S S)-7 \mathbf{a}$
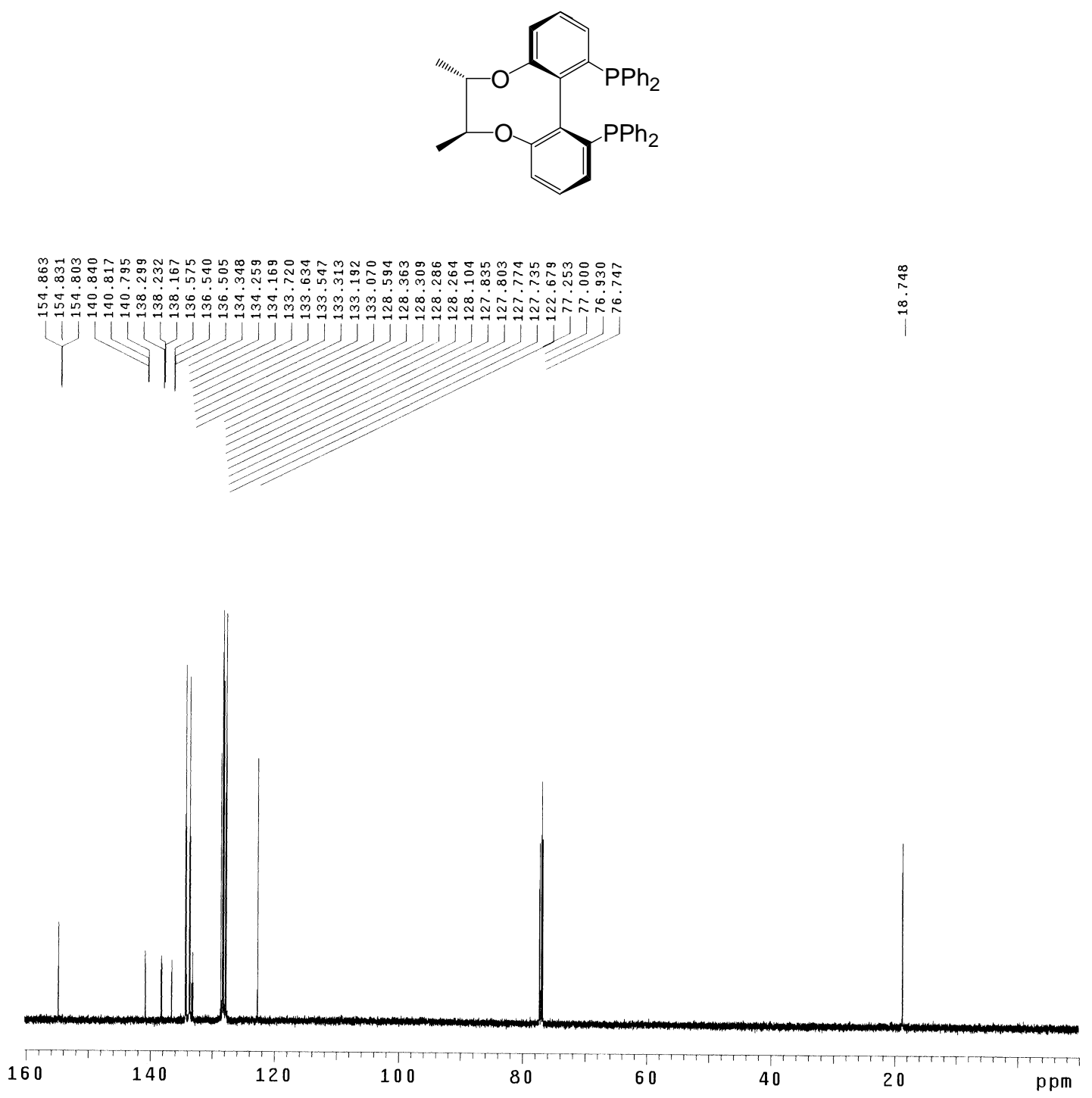
${ }^{1} \mathrm{H}$ NMR $\left(500 \mathrm{MHz}, \mathrm{CDCl}_{3}\right)$ of $(S R R)-7 \mathbf{b}$

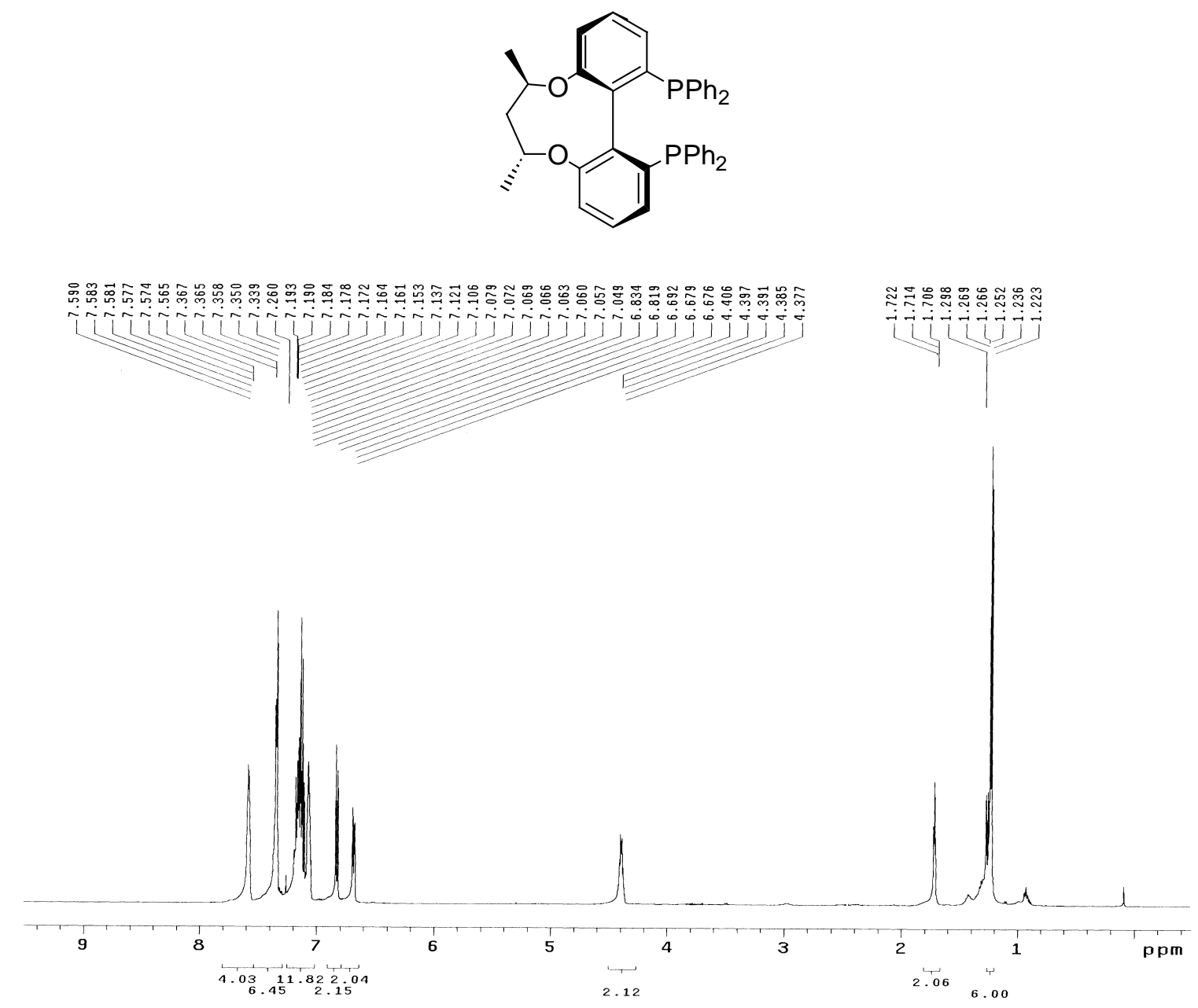


${ }^{31} \mathrm{P}$ NMR (202 MHz, $\left.\mathrm{CDCl}_{3}\right)$ of $(S R R)-7 \mathbf{b}$<smiles>C[C@@H]1COc2cccc(-c3ccccc3)c2-c2c(P)cccc2O1</smiles>

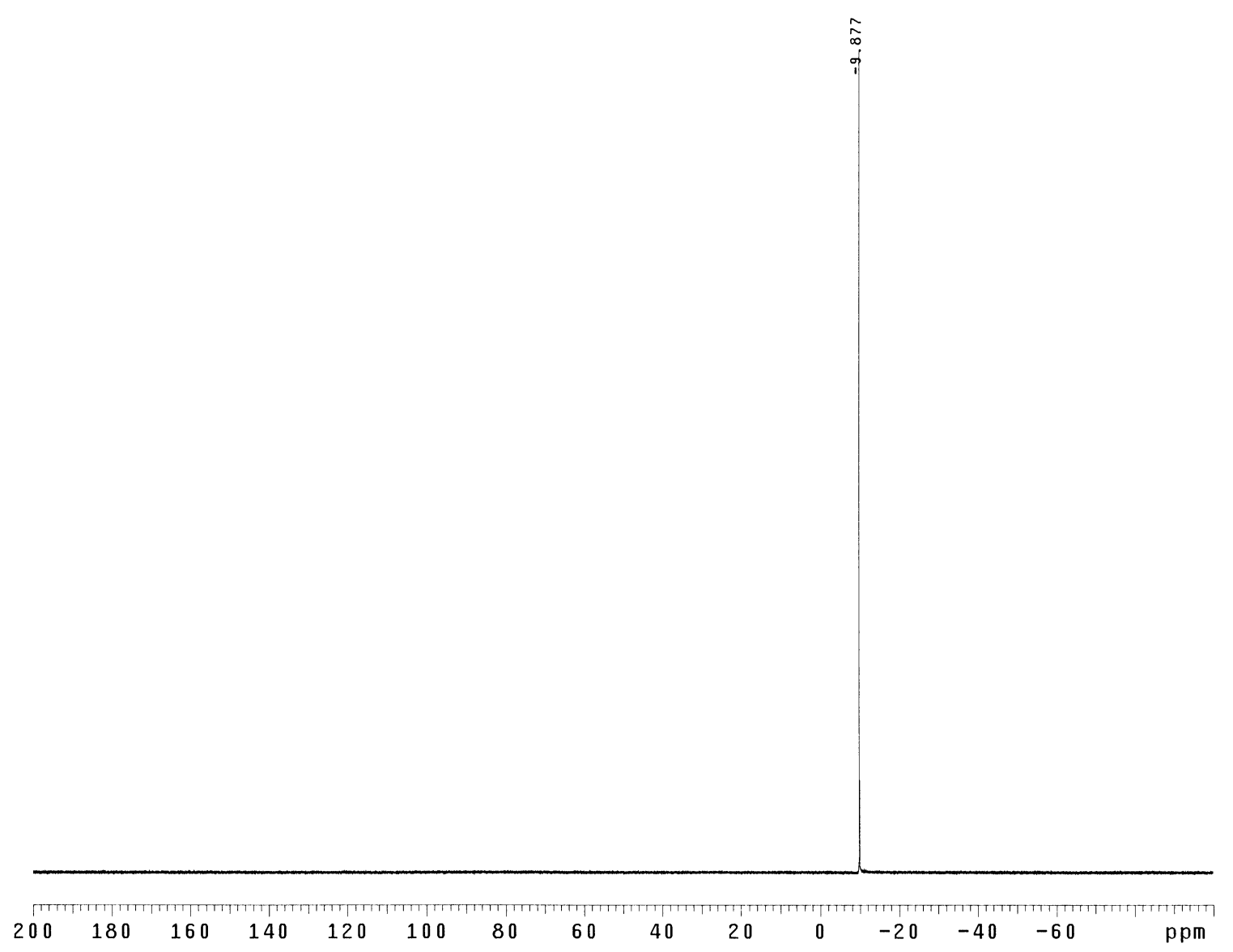


${ }^{13} \mathrm{C}$ NMR (125 MHz, $\left.\mathrm{CDCl}_{3}\right)$ of $(S R R)-7 \mathbf{b}$<smiles>CC(C)COc1cccc(P)c1-c1c(P)cccc1Pc1ccccc1</smiles>
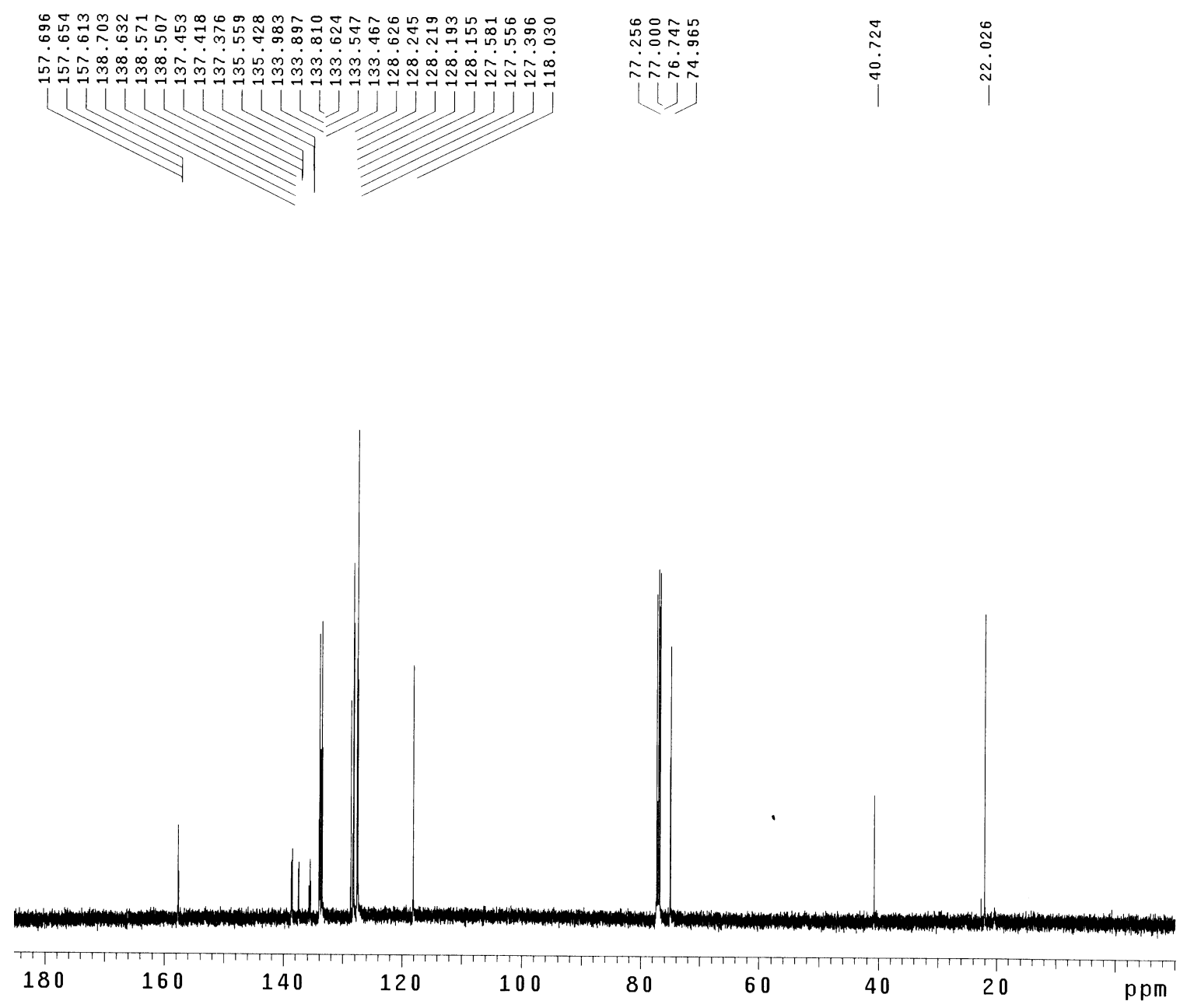
${ }^{1} \mathrm{H}$ NMR (500 MHz, $\left.\mathrm{CDCl}_{3}\right)$ of $(S R R)-7 \mathrm{c}$

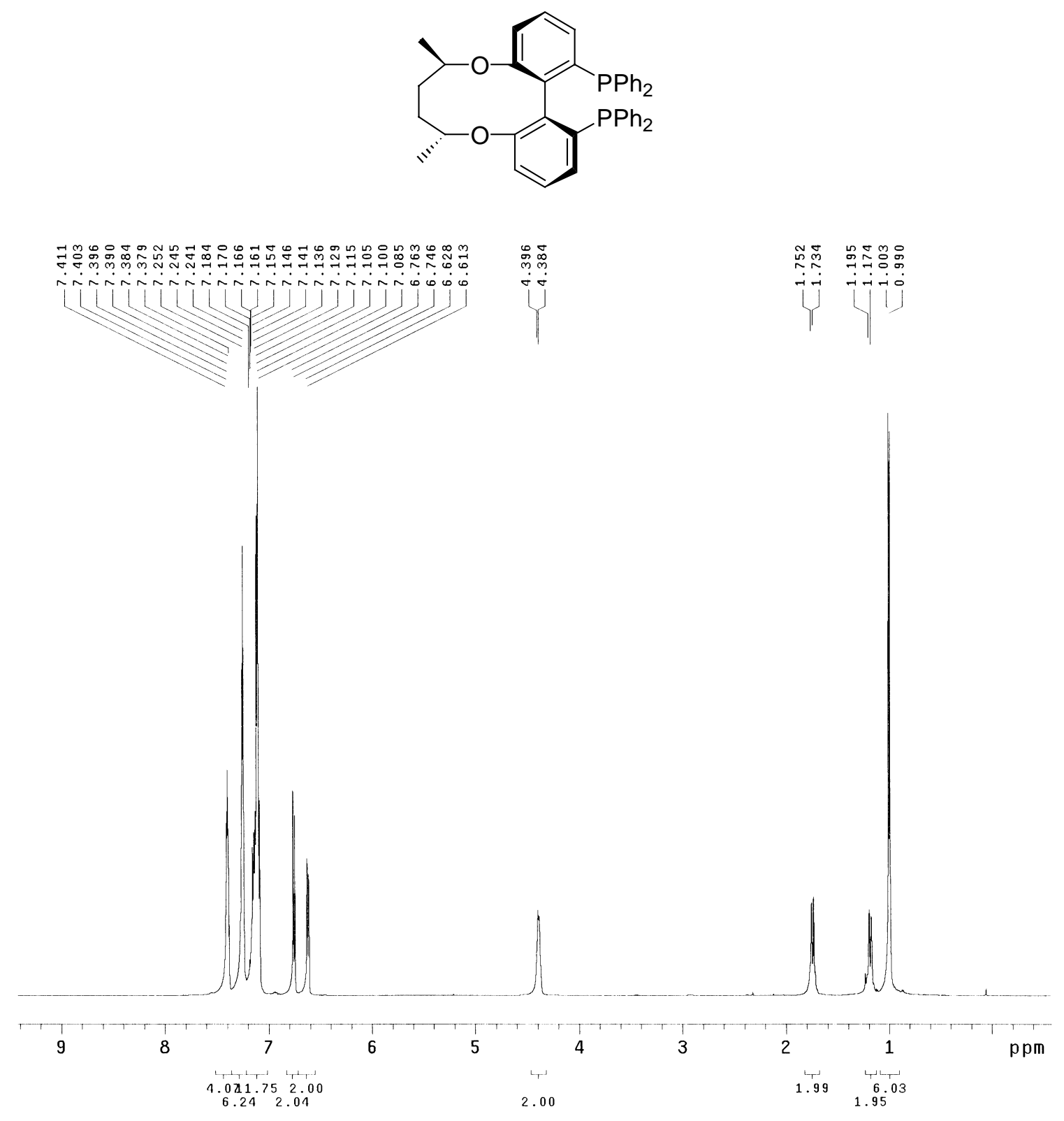


${ }^{31} \mathrm{P}$ NMR $\left(202 \mathrm{MHz}, \mathrm{CDCl}_{3}\right)$ of $(S R R)-7 \mathrm{c}$
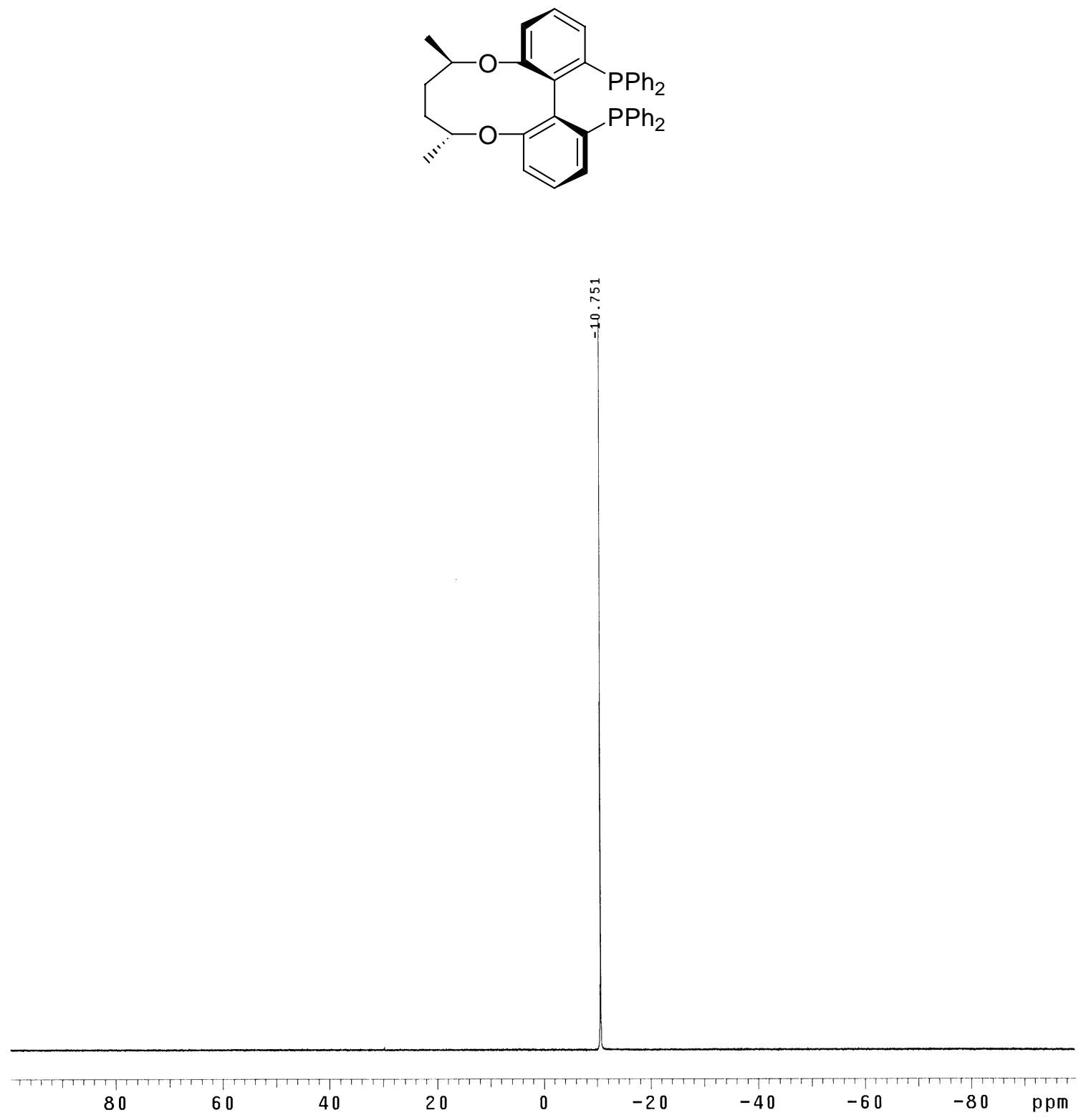
${ }^{13} \mathrm{C}$ NMR $\left(125 \mathrm{MHz}, \mathrm{CDCl}_{3}\right)$ of $(S R R)-7 \mathrm{c}$<smiles>C[C@@H]1CC[C@@H](C)Oc2cccc(-c3ccccc3)c2-c2c(cccc2-c2ccccc2)O1</smiles>

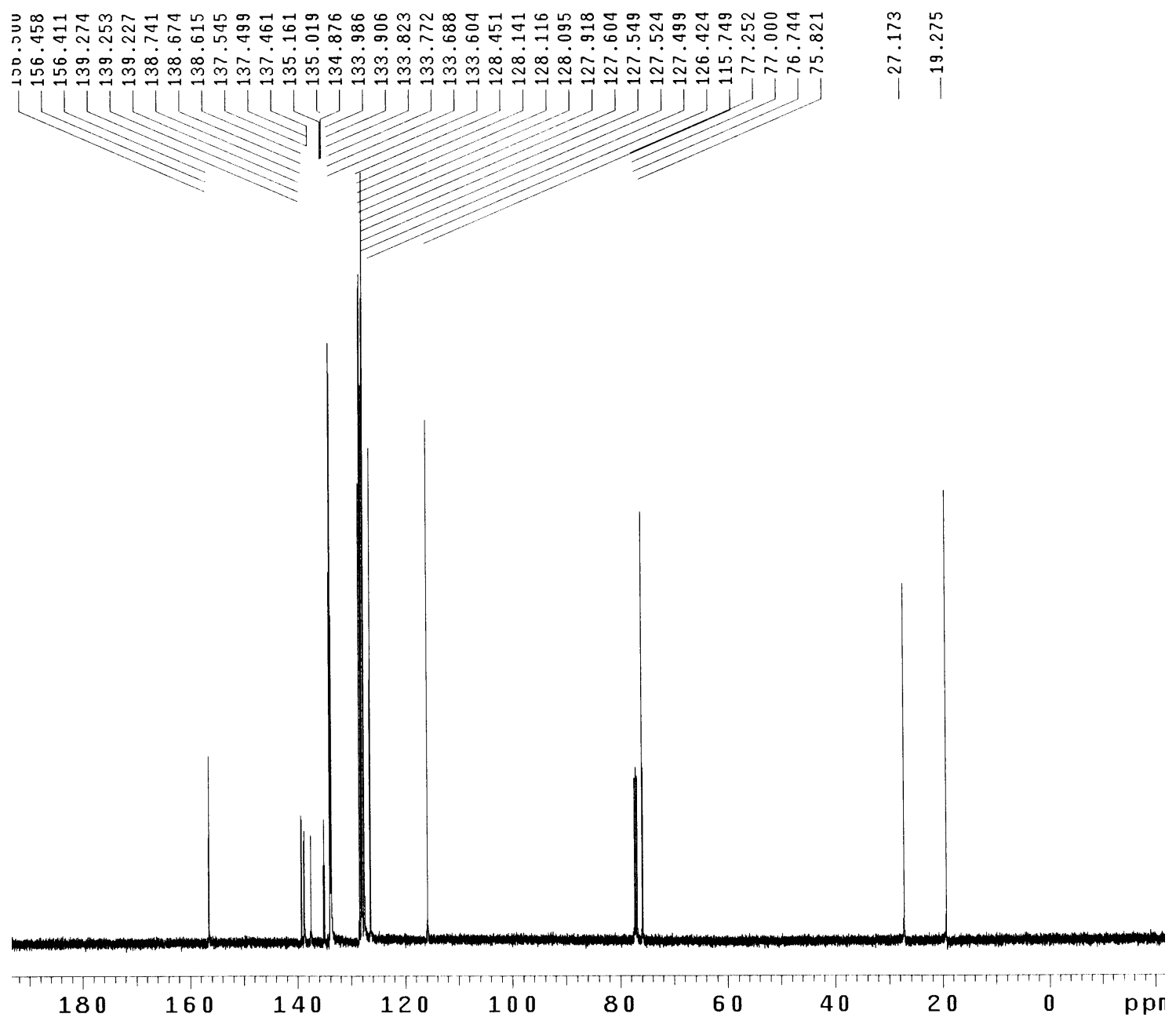


${ }^{1} \mathrm{H}$ NMR (500 MHz, $d$-DMSO) of (S)-9

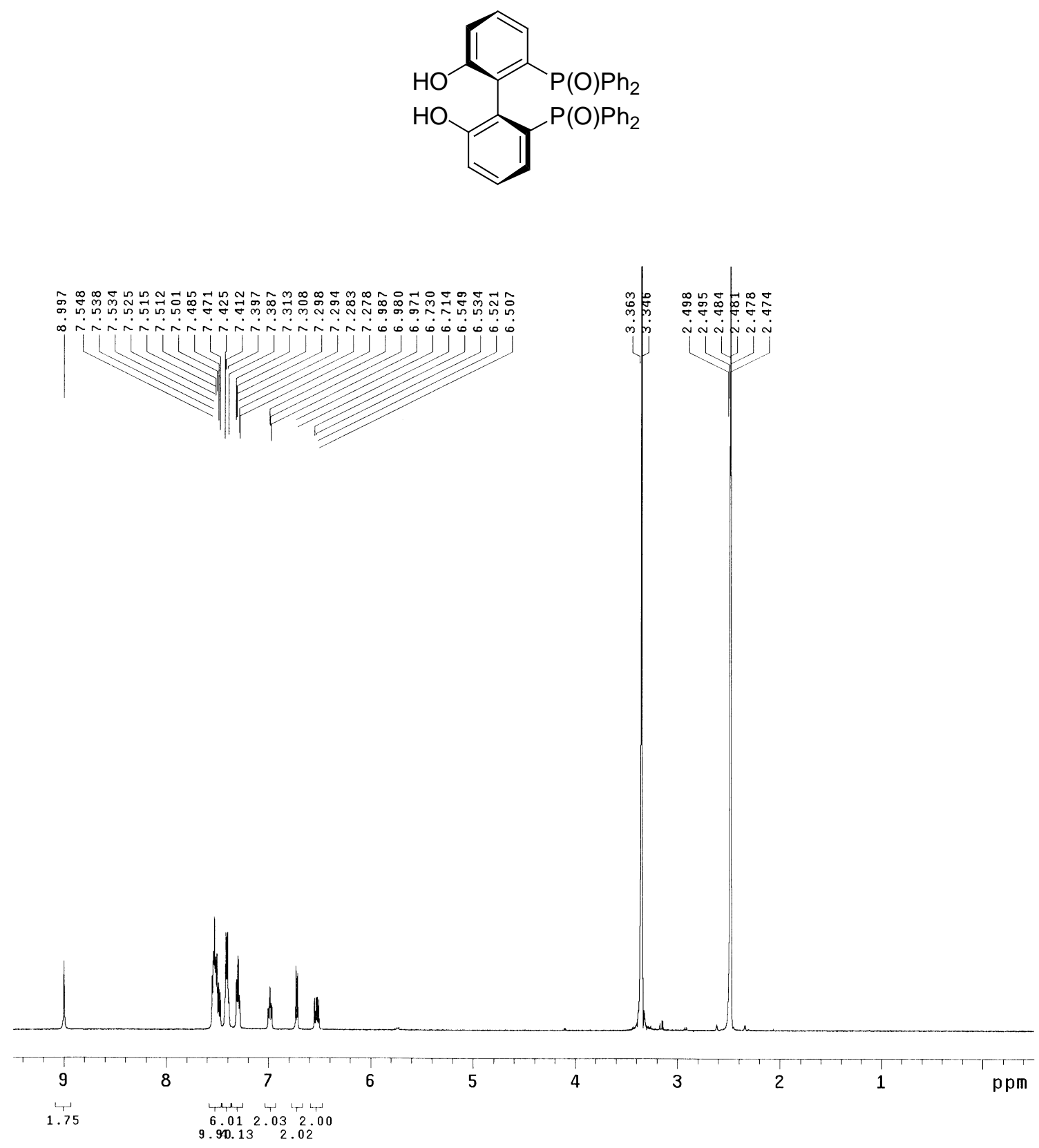


${ }^{31}$ P NMR (202 MHz, $d$-DMSO) of (S)-9<smiles>Oc1cccc(POc2ccccc2)c1-c1c(O)cccc1Pc1ccccc1</smiles>

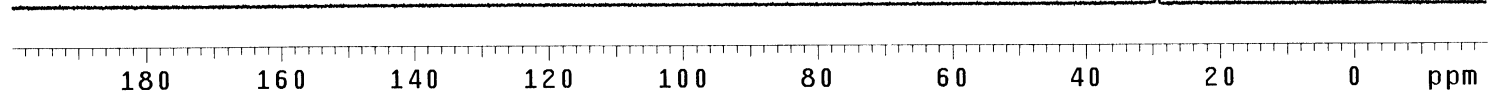




\section{${ }^{13} \mathrm{C}$ NMR (100 MHz, $\left.\mathrm{CD}_{2} \mathrm{Cl}_{2}+\mathrm{CD}_{3} \mathrm{OD}\right)$ of $(S)-9$}
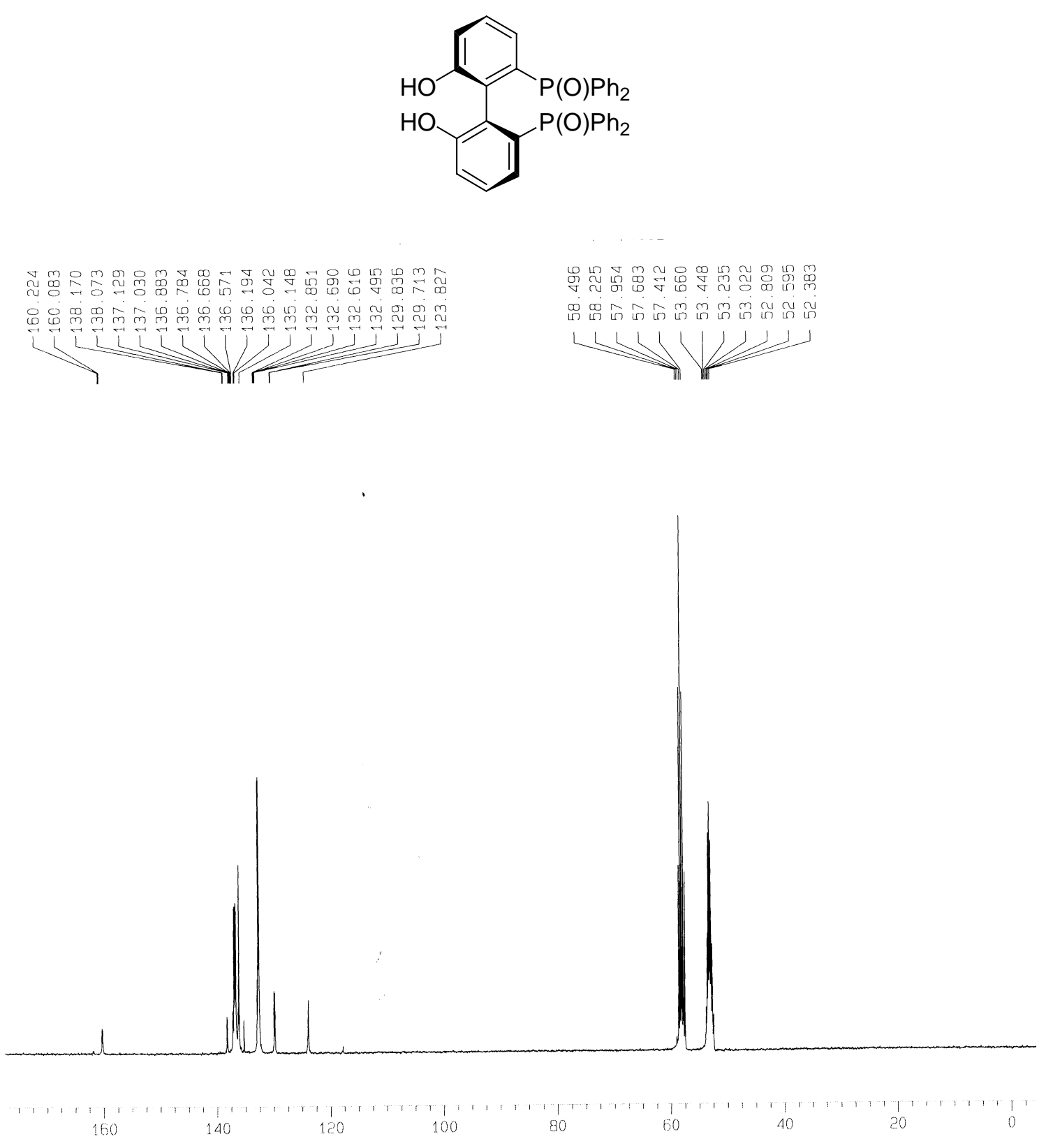
${ }^{1} \mathrm{H}$ NMR (500 MHz, $d$-DMSO) of (R)-9
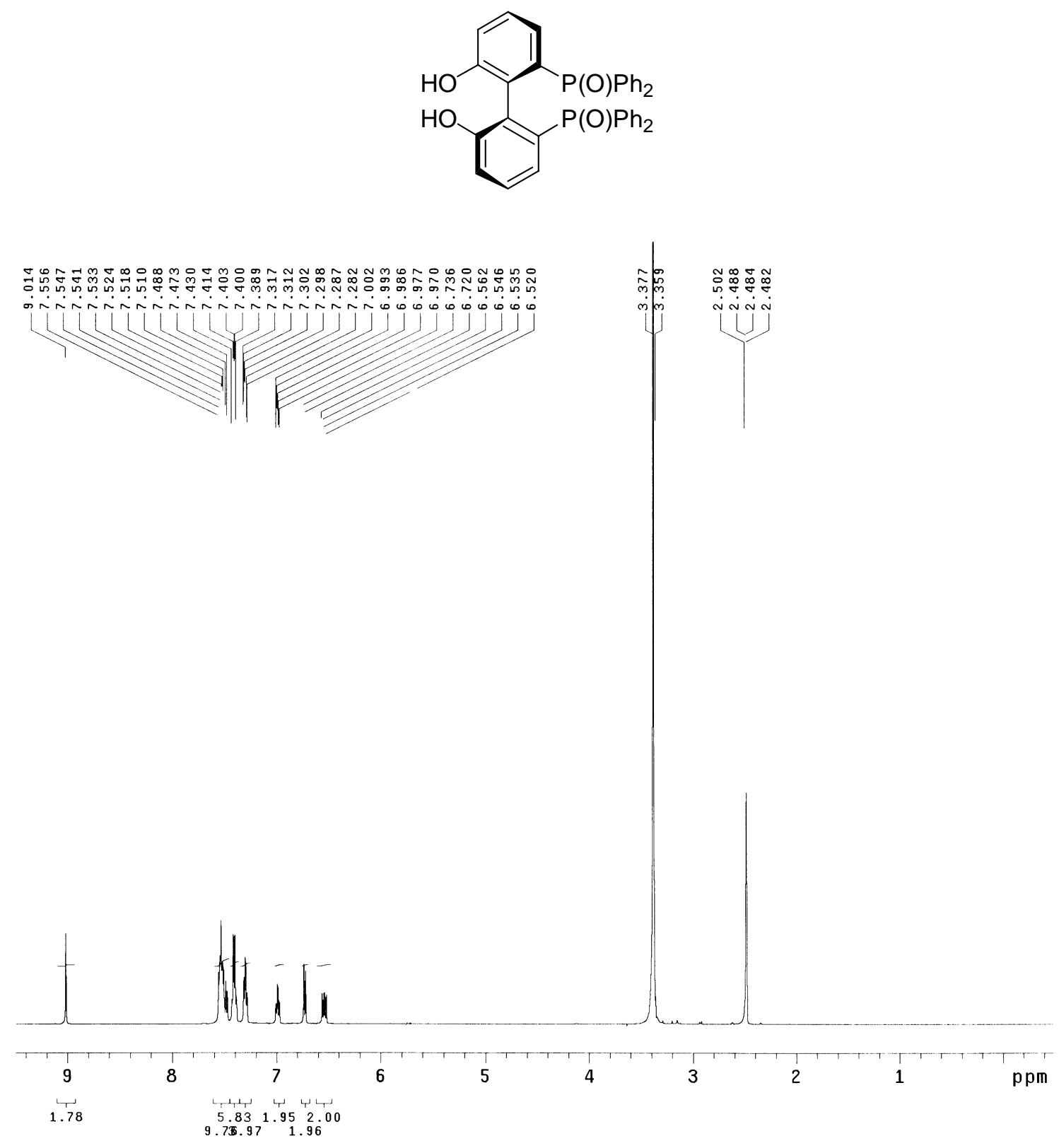
${ }^{31}$ P NMR (202 MHz, $d$-DMSO) of ( $R$ )-9
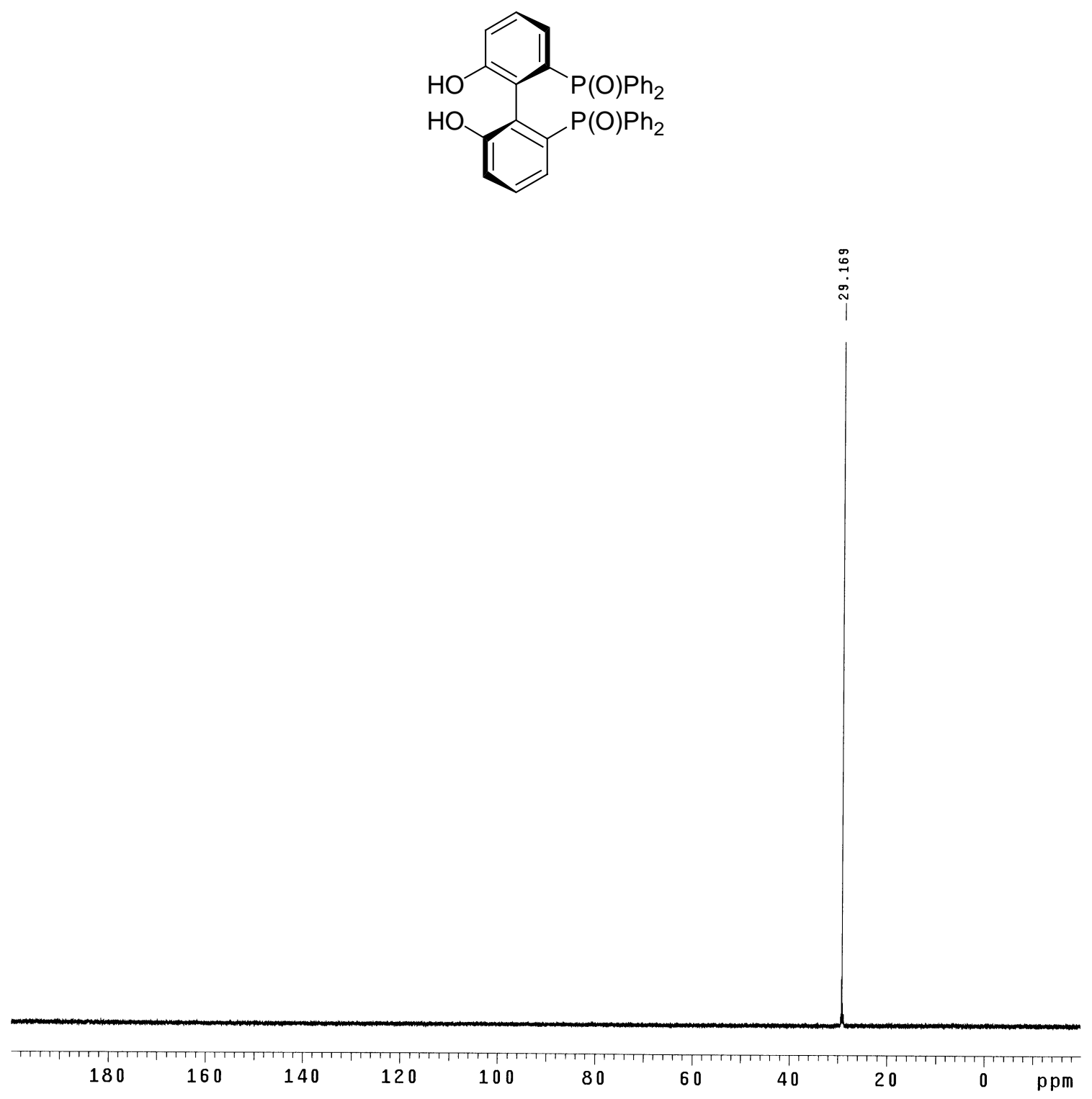


\section{${ }^{13} \mathrm{C}$ NMR (100 MHz, $\left.\mathrm{CD}_{2} \mathrm{Cl}_{2}+\mathrm{CD}_{3} \mathrm{OD}\right)$ of $(R)-9$}
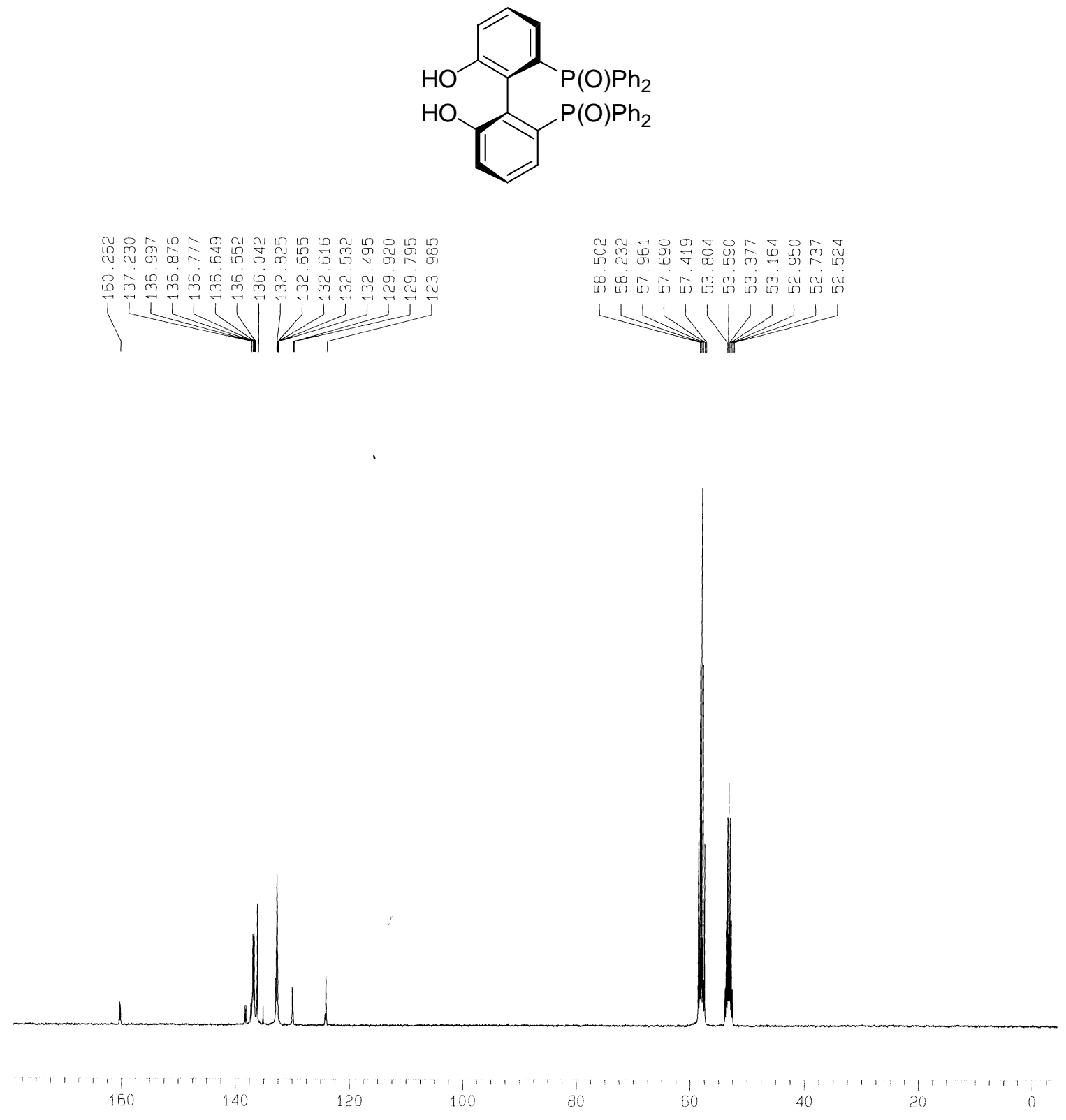